\title{
Theoretical Study of Electronic and Optical Properties in Doped Quantum Structures with Razavy Confining Potential: Effects of External Fields
}

\author{
Hassen Dakhlaoui ${ }^{1,2}$, J. A. Gil-Corrales ${ }^{3}$, A. L. Morales ${ }^{3}$, E. Kasapoglu ${ }^{4}$, A. Radu ${ }^{5}$, \\ R. L. Restrepo ${ }^{6}$, V. Tulupenko ${ }^{3}$, J. A. Vinasco ${ }^{3}$, M. E. Mora-Ramos ${ }^{7}$, and C. A. Duque ${ }^{3 *}$ \\ ${ }^{1}$ Nanomaterials Technology unit, Basic and Applied Scientific Research Center (BASRC), \\ College of Science of Dammam, Imam Abdulrahman Bin Faisal University, \\ P. O. Box 1982, 31441 Dammam, Saudi Arabia \\ ${ }^{2}$ Department of Physics, College of Sciences for Girls, \\ Imam Abdulrahman Bin Faisal University, Saudi Arabia \\ ${ }^{3}$ Grupo de Materia Condensada-UdeA, Instituto de Física, Facultad de Ciencias Exactas y Naturales, \\ Universidad de Antioquia UdeA, Calle 70 No. 52-21, Medellín, Colombia \\ ${ }^{4}$ Faculty of Science, Department of Physics, Sivas Cumhuriyet University, 58140 Sivas, Turkey \\ ${ }^{5}$ Department of Physics, "Politehnica" University of Bucharest, 313 Splaiul Independen, tei, 060042 Bucharest \\ ${ }^{6}$ Universidad EIA, Envigado 055428, Colombia and \\ ${ }^{7}$ Centro de Investigación en Ciencias-IICBA, Universidad Autónoma del Estado de Morelos, \\ Av. Universidad 1001, Cuernavaca 62209, Morelos, Mexico
}

(Dated: September 8, 2021)

\begin{abstract}
We investigate the energy states of confined electrons in doped quantum structures with Razavylike confining potentials. The theoretical investigation is performed within the effective mass and parabolic band approximations, including the influence of externally applied electric and magnetic fields. First, we analyze the case of a Razavy quantum well and determine its conduction subband spectrum, focusing on the lowest energy levels and their probability densities. These properties have been numerically determined by self-consistently solving the coupled system of Schrödinger, Poisson, and charge neutrality equations. Doping is introduced via an on-center $\delta$-like layer. In order to evaluate the associated total (linear plus nonlinear) optical absorption coefficient (TOAC), we have calculated the corresponding diagonal and off-diagonal electric dipole matrix elements, the main energy separation, and the occupancy ratio which are the main factors governing the variation of this optical response. A detailed discussion is given about the influence of doping concentration as well as electric and magnetic fields, which can produce shifts in the light absorption signal, towards either lower or higher frequencies. As an extension of the self-consistent method to a two-dimensional problem, the energy states of quantum wire system of circular cross section, with internal doping and Razavy potential have been calculated. The response of eigenvalues, self-consistent potentials and electron densities is studied with the variation of $\delta$-doping layer width and of the donor density. Finally, the origin of Friedel-like oscillations, that arise in the density profile, generated by the occupation of internal and surface electronic states has been explained.
\end{abstract}

\section{INTRODUCTION}

Semiconductor structures based on quantum wells (QWs), quantum-well wires , and quantum dots have acquired a huge importance in the process of designing low-dimensional devices, mostly due to their features of charge carriers confinement. To satisfy the exigence required by new generation of optoelectronic devices, the involved semiconductor structures must be suitably selected upon the basis of their confinement potential geometry, their dimensions, and the possible influence of certain external physical factors. Among these factors, we can cite the insertion of delta doped layers, as well as the application of either nonresonant intense laser field radiation, electric fields, magnetic fields, or a combination of these probe fields. All of them would play a crucial

${ }^{*}$ Corresponding author (C. A. Duque): carlos.duque1@udea.edu.co role in tuning the energy spectrum of the confined carrier states $[1-10]$.

As it is well known, the application of an external electric field leads to a tilt of the confining potential and pushes the electron wave functions towards the edge of the structure, producing a significant change in the energy levels and, per consequent, in the transition energies. This, in turn, produces noticeable modifications in the optical absorption response of the system. The application of a magnetic field provides an additional parabolic confinement that causes further spatial spread in the wave functions so that affecting the dipole matrix elements. The influences of both electric and magnetic fields have been theoretically and experimentally studied by many researchers [11-21]. For instance, Dakhlaoui et al., investigated the effects of magnetic and electric fields on the TOAC [22]. They showed that these probes can control the red and blue shifts of the TOAC in double and triple $\delta$-doped GaAs semiconductor heterostructures. Ungan et al. reported on the optical responses 
in hyperbolic-like QWs under external electric and magnetic fields [23]. They demonstrated that the TOAC and the total relative refractive index change coefficient can be shifted towards the blue or the red by adjusting the applied field intensities. The impacts of external perturbations on the optical and magnetic properties of GaAs/AlGaAs semi-parabolic QW have been widely discussed and commented by Hien et al. [24]. They proved that the magneto-optical properties are largely affected by the external fields.

Besides the application of external fields, the $\delta$-doping technique represents an ultimate concept in semiconductor's processing [25-31]. It is proved among the last years that this method is highly practical to adjust the energy levels in order to obtain the desired electronic mobility and optical absorption. For instance, in GaAs-based systems, the most typical n-type $\delta$-doping technique consists of inserting a thin layer containing silicon atoms. Once these atoms are ionized, they supply additional free electrons into the system. In addition, the silicon layer creates a triangular-shaped quantum well which would affect the spatial spread of the wave functions and furnish further confinement to the carriers [32, 33]. Various research works have explored, both experimentally and theoretically, the impact of $\delta$-doping on the behavior of the TOAC in semiconductor quantum nanostructures [3441]. For instance, Gaggero-Sager et al. studied the effects of temperature on the energy levels in a single doped QW [42], whereas Dhafer et al. showed the importance of an inserted $\delta$-InGaAs layer in a single $\mathrm{Al}_{x} \mathrm{Ga}_{y} \mathrm{In}_{1-x-y} \mathrm{As}$ QW. They found that the inserted layer can improve the intensity of the optical gain which is a requirement for fiber-optical communications [43]. J. Osvald studied the effect of a non-central $\delta$-doping layer on the energy levels and electronic density in GaAs QWs [44]. On the other hand, in 1980 M. Razavy used double potential wells in the quantum theory of molecules to describe the motion of a particle in the presence of two force fields [45]. These types of potentials are known today as Razavy potentials [46, 47], and are used as a model to describe the coupling of two molecules or quantum dots [48-51]. Effects of intense laser field and position dependent effective mass in Razavy QWs (Razavy-like quantum wells) were investigated in Ref. [52]. In their works, these authors have shown that the intensity of the TOAC can be largely tuned by varying the ionized impurity concentration in the doping layer.

Another class of heterostructures that can be studied by a similar procedure are quantum wires (QWRs), which are semiconductor structures in which electrons are confined in the transverse plane and therefore can only move in one dimension. A consequence of the above is that the electronic energy levels present a discrete behavior along the cross section of the structure, while in the free dimension the electrons present a continuous spectrum. QWRs have been the subject of study for more than 40 years, as noticed -for example- from a bunch of early references [53-57]. Due to the remarkable applications of this type of low-dimensional structures in areas as diverse as flexible electronics (when designing thin-film transistors) or high-efficiency solar cells [58], among others; to seek an improvement in the properties of electronic conduction would become a boost to development and characterization of this type of systems. In recent years, results of great interest have been reported along these lines, some of which have appeared in references [59-62].

Motivated by all the cited works, here we aim to investigate the effects of the concentration of an on-center thin doping layer and of externally applied electric and magnetic fields on the TOAC in Razavy-like GaAs quantum structures. We shall study the impact of these parameters on the lowest energy separations, the occupancy ratios, and dipole matrices which are preponderant factors governing the TOAC variation. In the first part we develop the one-dimensional problem of a GaAs $\delta$ doped QW (with $z$-oriented growth direction) having a Razavy-type confinement potential. For this system, the confined electronic states are calculated, as well as the self-consistent potentials, with a special care taken on determining the Fermi level position, affected by the both temperature and ionized impurity distribution. Subsequently, external electric and magnetic fields are applied in order to analyze the variations in obtained results and finally the optical absorption response is investigated. The second problem dealt with is a two-dimensional system corresponding to a GaAs QWR with circular cross section and exposed borders (the confinement plane has been taken as $x y$ ), with an additional $\delta$-type doping and an inner Razavy-like potential. The problem for the electron energy states in this case is also solved in a selfconsistent way, taking into account a fundamental difference: In this case the Fermi level is no longer modified by the density of donors because the system has exposed borders. So, a "Fermi Level Pinning" is presented which keeps it fixed. Another fundamental difference with respect to the QW structure is that, in this type of systems, variations in the electron density profile known as Friedel oscillations can occur at low temperatures. To study this particular phenomenon, electron states in this system has been calculated for $T=10 \mathrm{~K}$ and not at $300 \mathrm{~K}$ as the first problem. In this second case, the electronic states, self-consistent potential and electron density have been calculated for different widths of the $\delta$-layer, with different concentrations. Self-consistent procedures have been performed by numerically solving the effective mass conduction band equation using the finite element method (FEM). In accordance, this work is organized as follows: in sections 2 we outline the theoretical equations and the method of resolution for the one-dimensional system quantum well and the quantum wire system with exposed boundaries. The discussion and comments on the obtained results for each system are presented in section 3, while the conclusions are given in section 4 . 


\section{THEORETICAL FRAMEWORK}

\section{A. Razavi quantum-well (quantum well with Razavy-like potential)}

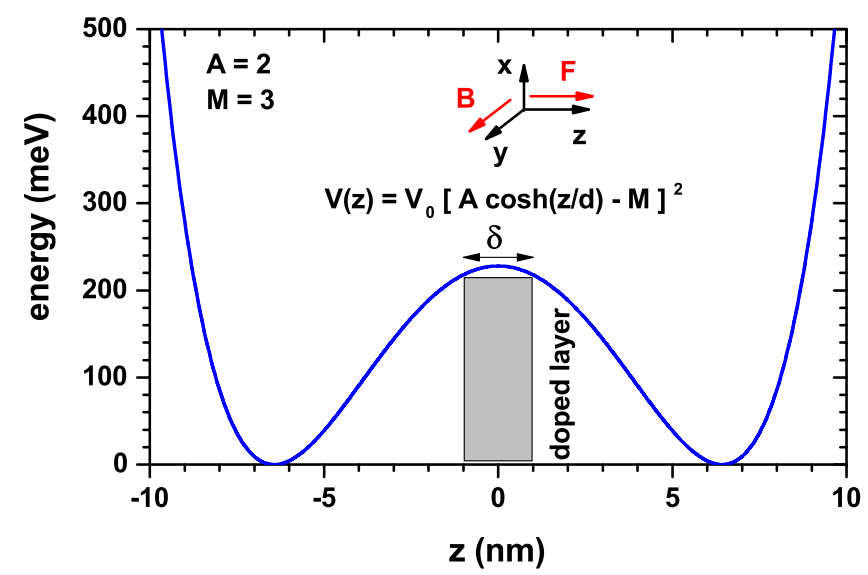

FIG. 1: (color online) Schematic representation of doped Razavy quantum well under applied electric and magnetic fields.

The problem under consideration here consists of a GaAs conduction electron that moves under the influence of an on-center doped Razavy-like QW potential and undergoes the effect of external electric and magnetic fields. The electric field is assumed to be oriented along the $z$-growth direction and the magnetic field is applied perpendicular to the electric field, and lies within the plane of the layers. In Fig. 1 we plot a Razavy-like confining profile, together with a schematic representation of the n-type doped layer. The $\delta$-parameter (2-nm in this work) corresponds to the finite width of the on-center doped layer, which has a two-dimensional $N_{d}$ concentration of ionized donor atoms. Within the effective mass and parabolic band approximations, the Hamiltonian for a confined electron is given by [1]:

$$
H=\frac{1}{2 m^{*}}\left[\vec{p}+\frac{e}{c} \vec{A}(\vec{r})\right]^{2}+V_{c}(z)+V_{H}(z)+e F z,
$$

where $c, e$, and $m^{*}$ denote the speed of light in the vacuum, the absolute value of the elementary charge, and the electron effective mass, respectively. Additionally, $\vec{p}$ represents the momentum operator, and $F$ is the intensity of the applied electric $(\vec{F})$. Within the Landau gauge, the vector potential associated to the applied magnetic field $(\vec{B})$ is given by $\vec{A}(\vec{r})=B z \hat{x}$ ( $B$ stands for the magnetic field intensity). Furthermore, $V_{H}(z)$ is the Hartree potential, which represents the additional conduction band reshaping due to the presence of the low-dimensional electron gas that arises from the donor ionization in the $\delta$ layer. Besides, $V_{c}(z)$ is the Razavy-like confining potential given by [52]:

$$
V_{c}(z)=V_{0}\left[A \cosh \left(\frac{z}{D}\right)-M\right]^{2},
$$

where a set of parameters with $V_{0}=228 \mathrm{meV}, A=2$, $M=3, D=\frac{L_{e f f}}{3}$, and $L_{e f f}=20 \mathrm{~nm}$ (the total width of the QW) has been chosen for the calculation.

The wave function associated to the Hamiltonian in Eq. (1) can be written as [63]:

$$
\psi(\vec{r})=\exp \left(i \overrightarrow{k_{\perp}} \cdot \vec{\rho}\right) \Phi^{\prime}(z),
$$

where $\overrightarrow{k_{\perp}}=\left(k_{x}, k_{y}\right), \vec{\rho}=(x, y)$, and $\Phi^{\prime}(z)$ satisfies the following differential equation [63]:

$$
H^{0} \Phi^{\prime}(z)=\left(E_{z}-\frac{\hbar^{2} k_{y}^{2}}{2 m^{*}}\right) \Phi^{\prime}(z) .
$$

where

$$
\begin{aligned}
H^{0}=-\frac{\hbar^{2}}{2 m^{*}} \frac{d^{2}}{d z^{2}} & +\frac{e^{2} B^{2}}{2 m^{*}}\left(z+\frac{\hbar k_{x}}{e B}\right)^{2} \\
& +e F\left(z+L_{e f f} / 2\right)+V_{H}(z) .
\end{aligned}
$$

The $z$-component of the Eq. (4) can be written in the form [63]:

$$
H \Phi(z)=E_{z} \Phi(z)
$$

where

$$
\begin{aligned}
H=-\frac{\hbar^{2}}{2 m^{*}} \frac{d^{2}}{d z^{2}} & +\frac{e^{2} B^{2}}{2 m^{*}} z^{2} \\
& +e F\left(z+L_{e f f} / 2\right)+V_{H}(z) .
\end{aligned}
$$

Note that the solutions of Eq. (6) correspond to the bottom of the confined conduction subbands, i.e., $\left(k_{x}, k_{y}\right)=$ $(0,0)$.

The calculation of the Fermi level $\left(E_{F}\right)$ is based on the charge neutrality condition, such that the total number of electrons must be equal to the total number of ionized donors per unit area (assuming that all the silicon atoms are ionized)

$$
n_{d}=\sum_{i} \frac{m^{*} k_{B} T}{\pi \hbar^{2}} \log \left[1+\exp \left(\frac{E_{F}-E_{i}}{k_{B} T}\right)\right]
$$

where $k_{B}$ is the Boltzmann constant and $T(=300 \mathrm{~K}$ in this work) denotes the absolute temperature.

The Hartree potential $V_{H}(z)$ which describes the electrostatic interaction of electrons and ionized ions results from the solution of the generalized Poisson equation [64]:

$$
\frac{d^{2} V_{H}(z)}{d z^{2}}=\frac{e^{2}}{\varepsilon \varepsilon_{0}}\left[n_{d}(z)-n(z)\right],
$$

where $\varepsilon\left(\varepsilon_{0}\right)$ is the GaAs (vacuum) static dielectric constant and $n_{d}$ is the $3 \mathrm{D}$ donor density in the delta layer. Also, in Eq. (9)

$$
n(z)=\sum_{i} \frac{m^{*} k_{B} T}{\pi \hbar^{2}} \log \left[1+\exp \left(\frac{E_{F}-E_{i}}{k_{B} T}\right)\right] \Phi_{i}^{2}(z),
$$


The Eqs. (6-9) are discretized using the finite difference method (FDM) and solved iteratively. Under such a procedure, the Schrödinger and Poisson equations are then changed to matrices of type $A x=\lambda x$ and $B x=\rho$, respectively. Here, $\lambda$ represents the energy and $x$ is a column wave vector describing the electron wave function. After computing the energy levels and their corresponding wave functions, the linear, third-order nonlinear, and total optical absorption coefficients for the intersubband transitions between initial and final states $E_{i} \rightarrow E_{f}$ can be evaluated from [65-67]:

$$
\begin{gathered}
\alpha^{1}(\omega)=\omega \sqrt{\frac{\mu}{\varepsilon_{R}}} \times \frac{e^{2}\left|M_{i f}\right|^{2} \widetilde{\sigma}_{i f} \hbar / \tau_{i n}}{(\Delta E-\hbar \omega)^{2}+\left(\hbar / \tau_{i n}\right)^{2}}, \\
\alpha^{3}(\omega, I)=-2 \omega \sqrt{\frac{\mu}{\varepsilon_{R}}}\left(\frac{I}{\varepsilon_{0} n_{r} c}\right) \\
\times \frac{e^{4}\left|M_{i f}\right|^{4} \widetilde{\sigma}_{i f}\left(\hbar / \tau_{i n}\right)}{\left[(\Delta E-\hbar \omega)^{2}+\left(\hbar / \tau_{i n}\right)^{2}\right]^{2}} \\
\times\left(1-\Omega \frac{(\Delta E-\hbar \omega)^{2}-\left(\hbar / \tau_{i n}\right)^{2}+2 \Delta E(\Delta E-\hbar \omega)}{(\Delta E)^{2}+\left(\hbar / \tau_{i n}\right)^{2}}\right)
\end{gathered}
$$

and

$$
\alpha(\omega, I)=\alpha^{1}(\omega)+\alpha^{3}(\omega, I) .
$$

In the previous equations $\Omega=\frac{\left|M_{f f}-M_{i i}\right|^{2}}{\left|2 M_{i f}\right|^{2}}, \Delta E=E_{f}-$ $E_{i}$,

$$
M_{i f}=\int_{-\infty}^{+\infty} \Phi_{f}^{*}(z) \Phi_{i}(z) z d z
$$

is the reduced dipole matrix element, and $\widetilde{\sigma}_{i f}=$ $\frac{m^{*} k_{B} T}{L_{e f f} \pi \hbar^{2}} \sigma_{i f}$, with

$$
\sigma_{i f}=\ln \left\{\frac{1+\exp \left[\left(E_{F}-E_{i}\right) / k_{B} T\right]}{1+\exp \left[\left(E_{F}-E_{f}\right) / k_{B} T\right]}\right\} .
$$

Here, $\mu$ represents the free space permeability, $\tau_{i n}=$ $0.14 \mathrm{ps}$ stands for the intersubband relaxation time, and $I$ is the intensity of incident light. The other physical parameters used in this work are [68, 69]: $m^{*}=0.067 m_{0}$ (where $m_{0}$ is free electron mass), $e=1.602 \times 10^{-19} \mathrm{C}, \hbar=$ $1.056 \times 10^{-34} \mathrm{~J} \mathrm{~s} ; n_{r}=\sqrt{\varepsilon}=3.2, \mu=4 \pi \times 10^{-7} \mathrm{H} \mathrm{m}^{-1}$, $\varepsilon=12.35, \varepsilon_{0}=8.854 \times 10^{-12} \mathrm{C}^{2} \mathrm{~N}^{-1} \mathrm{~m}^{-2}, \varepsilon_{R}=\varepsilon \varepsilon_{0}$, and $I=0.5 \mathrm{MW} / \mathrm{cm}^{2}$.

\section{B. Razavi quantum-wire}

The theory required to study the two-dimensional problem of a QWR with delta-type doping is very similar to that developed for the one-dimensional well system since both problems are solved by means of the selfconsistent method. For this reason, and to clarify the procedure without being redundant, some equations have been rewritten in this section, with the aim of presenting generalization to a $2 \mathrm{D}$ system.

The structure under consideration corresponds to an infinite QWR of GaAs with circular cross section and exposed borders. That is, the system is not immersed in any substrate but is in contact with vacuum. Additionally, a Razavy potential and a delta-doped layer have been added to shape the confining potential. A consequence of the above is the appearance of both inner and surface states in the system, giving rise to the phenomenon known as "Fermi Level Pinning" [70] in which a charge transfer from bulk states to surface states occurs, thus causing the Fermi level to remain fixed at a value within the band gap, regardless of the donor density in the system. For GaAs, a potential value of 0.7 $\mathrm{eV}$, which corresponds to half the gap is normally used for the surface.

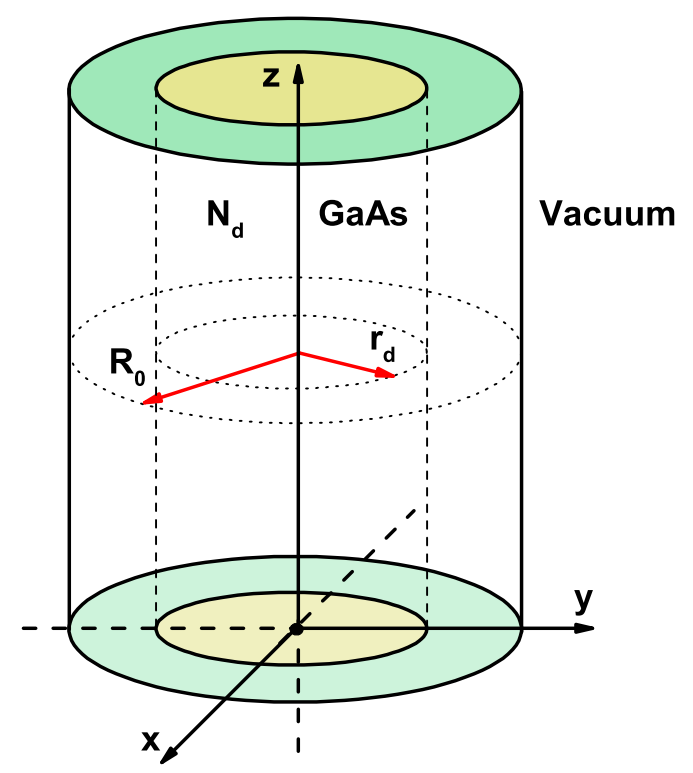

FIG. 2: Diagram of the infinite quantum wire of GaAs surrounded by vacuum, with a circular cross section of radius $R_{0}$. The coordinate system is centered on the symmetry axis of the wire. An inner doping layer of radius $r_{d}$ has been included in the system.

Figure 2 shows the schematic representation of the quantum wire, as well as the coordinate system used for numerical calculations. This is a QWR of radius $R_{0}$, surrounded by vacuum. Inserted at the center of this cylinder, an inner layer of -fully- ionized donors of radius $r_{d}$ is included.

Again, the chosen numerical approach to solve this type of problem is the self-consistent method. It consists of a bidirectional coupling between the Poisson and Schrödinger equations. The electric potential that comes from the Poisson equation (including the effect of donor density) must be added to the confining potential term to later be included in the Schrödinger equation. Similarly, a statistically weighted sum of the probability density as- 
sociated with the electronic occupation of the states of the Schrödinger problem contributes to the space charge density that enters the Poisson equation. This process implies an iteration scheme where the confining potential becomes modified by the redistribution of charges.

As a starting point of the self-consistent method, and following the Luscombe and Luban procedure [71], we shal consider that electrons in the cross section of the QWR behave as a two-dimensional charge-carrier gas. This indicates that a good approximation for the electron density would be the Thomas-Fermi one (Eq. 16). Such electron density must contribute to the charge density in the system and therefore to the Hartree potential that is obtained through the Poisson equation,

$$
n(x, y, T)=N_{C} F_{1 / 2}\left(\beta\left(E_{F}-\Upsilon(x, y, T)\right)\right)
$$

where $\beta=1 / k_{B} T$ is the Boltzmann factor, $N_{C}=$ $\left(2 m^{*} / \pi \beta \hbar^{2}\right)^{3 / 2} / 4$ is the effective density of states, $\Upsilon(x, y, T)$ is the electronic potential generated by the Fermi level pinning on the exposed lateral surface, the level of doping and the lateral dimensions of the system and is given by $\Upsilon(x, y, T)=-e \phi(x, y, T)$, where $\phi(x, y, T)$ is the Hartree potential. Besides, $e$ is the charge of the electron, $m^{*}$ is the effective mass, and $F_{1 / 2}(x)$ is the well known Fermi-Dirac integral. With the electron density given by Eq. 16 and the density of donors $N_{d}$ given by $N_{d}$ for $r \leq r_{d}$ and 0 for $r>r_{d}$, it is possible to write the expression for the charge density in the system as,

$$
\rho(x, y, T)=e\left(N_{d}-n(x, y, T)\right)
$$

where $\epsilon_{r}$ and $\epsilon_{0}$ are the relative permittivity and vacuum permittivity respectively. Note that, unlike the QW, now the charge density has a two-dimensional dependence, in the same way the donor density for this case is identified as $N_{d}$ (for the well system it is labeled as $n_{d}$ ). This charge density must enter the two-dimensional Poisson equation (Eq. 18) to obtain the Hartree potential,

$$
-\epsilon_{0} \epsilon_{r} \nabla^{2} \phi(x, y, T)=\rho(x, y, T)
$$

This equation should be solved taking into account the boundary conditions imposed by the Fermi level pinning, which for GaAs takes the form $\phi(\Omega)=-\left(E_{F}+0.7 \mathrm{eV}\right) / e$, where $\Omega$ represents the QWR boundary. The potential, $\phi(x, y, T)$, obtained through Eq. 18 must contribute to the potential energy term in the Schrödinger equation,

$$
U(x, y, T)=-e \phi(x, y, T)+V_{c}(x, y)
$$

where $-e \phi(x, y, T)$ is the contribution that comes from the Poisson equation and the redistribution of charges due to doping, and $V_{c}(x, y)$ is the Razavy 2D potential, which has the same form as Eq. 2, changing $z$ for $\sqrt{x^{2}+y^{2}}$. In this case, $V_{0}=0.228 \mathrm{eV}, A=2, M=3$, $D=1.57 \mathrm{~L}$, and $L=20 \mathrm{~nm}$. On the other hand, the electrons are assumed to be totally confined within the volume of the QWR and, therefore, it must be satisfied that $\Psi(\Omega)=0$ in the Schrödinger equation,

$$
-\frac{\hbar^{2}}{2 m^{*}} \nabla^{2} \Psi_{i k}(\vec{r})+U(x, y, T) \Psi_{i k}(\vec{r})=E_{i k} \Psi_{i k}(\vec{r})
$$

Note that in Eq. 21 we have used the approximation of effective mass for electrons in GaAs. In this equation $\Psi(\vec{r})$, the wave function of the system, takes the form,

$$
\Psi_{i k}(\vec{r})=e^{i k z} \psi_{i}(x, y)
$$

Considering now the Schrödinger equation in the $x y$ plane, with eigenvalue $E_{i}$ associated with the state $\psi_{i}(x, y)$, we have to solve the problem

$$
-\frac{\hbar^{2}}{2 m^{*}} \nabla^{2} \psi_{i}(x, y)+U(x, y, T) \psi_{i}(x, y)=E_{i} \psi_{i}(x, y),
$$

with $\psi_{i}(\Omega)=0$. From here, it is possible to find the first set of eigenfunctions and eigenvalues for the system. With this set we can calculate the electron density associated with the occupation of the internal states in the system,

$$
\eta(x, y, T)=\xi \sum_{i=1} F_{-1 / 2}\left(\beta\left(E_{F}-E_{i}\right)\right)\left|\psi_{i}(x, y)\right|^{2}
$$

where $\xi=\sqrt[3]{4 N_{C}}$. This equation represents the density of electron gas at a point $(x, y)$ and temperature $T$. From the electron density calculated in Eq. 24, a new profile for the charge density of the system is obtained:

$$
\rho_{\text {new }}(x, y, T)=e\left(N_{d}-\eta(x, y, T)\right) .
$$

Replacing this charge density into Poisson's equation (18), a new Hartree potential $\phi_{n e w}(x, y, T)$ is obtained that will -again- contribute to the potential energy term in the Schrödinger equation. Then, a new set of eigenfunctions and eigenvalues for the system $\psi_{i}^{\text {new }}, E_{i}^{\text {new }}$ is obtained. This set will be associated to a new electron density profile $\eta_{\text {new }}$ relative to the occupation of each of each state of the system. In this way, the process is repeated iteratively until the absolute value of the difference between potential terms corresponding to two successive self-consistent steps is smaller than a certain tolerance $\left|U-U_{\text {old }}\right|<10^{-6} \mathrm{eV}$. When this condition is met, the system is said to have reached self-consistency. Note that the confining potential and the Razavy potential do not change with the iterative process, only the electrostatic potential changes due to the redistribution of the charge carriers. 
Once self-consistency has been reached, the final set of eigenvalues and eigenfunctions as well as the selfconsistent potential found are the correct solutions for the quantum wire with exposed boundaries, Razavy potential, and donor density $N_{d}$. Figure 3 shows the plots of first five QWR confined state wave functions for three different values of $r_{d}$; from left to right, each column corresponds to: $r_{d}=5 \mathrm{~nm}, r_{d}=10 \mathrm{~nm}$, and $r_{d}=15 \mathrm{~nm}$. Despite varying the inner radius $r_{d}$, all the figures maintain the same cross-sectional area since the outer radius $R_{0}=50 \mathrm{~nm}$ remains fixed. The electron density $N_{d}$ has been fixed as $3 \times 10^{19} \mathrm{~cm}^{-3}$ for all three cases, and the temperature has also been kept at $T=10 \mathrm{~K}$. The color scale in each figure goes from the blue, which corresponds to negative values of the wave function, to dark red which represents positive values. Yellow indicates the points at which the wave function is zero. With these parameters a degeneracy between $\psi_{1}$ and $\psi_{2}$ appears for all $r_{d}$. Note how for $r_{d}=5 \mathrm{~nm}$-first column plots- the state $\psi_{3}$ has the shape of an $s$-orbital, followed by the state $\psi_{4}$ which has a $d$-type orbital (in analogy with atomic orbitals). For values of $r_{d}=10 \mathrm{~nm}$ and $r_{d}=15 \mathrm{~nm}$, this $s$ state no longer appears in $\psi_{3}$, it takes the form of a $d$ state. This means that as long as the value of $r_{d}$ augments, an exchange between states of type $s$ and type $d$ is present for the third excited state.

On the other hand, the first row at the top of Fig. 3, corresponds to the ground state $\psi_{0}$ for each system, again in analogy with the atomic orbitals, a clear symmetry equivalent to an $s$-type state is evidenced. For all system configurations, it is readily apparent that there is a high probability of finding the electrons close to the center of gravity of the structure. Moreover, as $r_{d}$ increases, the probability at the center of the structure diminishes, at the time that increases along the radial direction. Then, the electrons tend to be distributed along the cross section with the increase of the doping region width. The first and second excited states, $\psi_{1}$ and $\psi_{2}$, are presented on the second and third rows from top to bottom. Note that these states present a symmetry similar to the $p$-type orbitals.

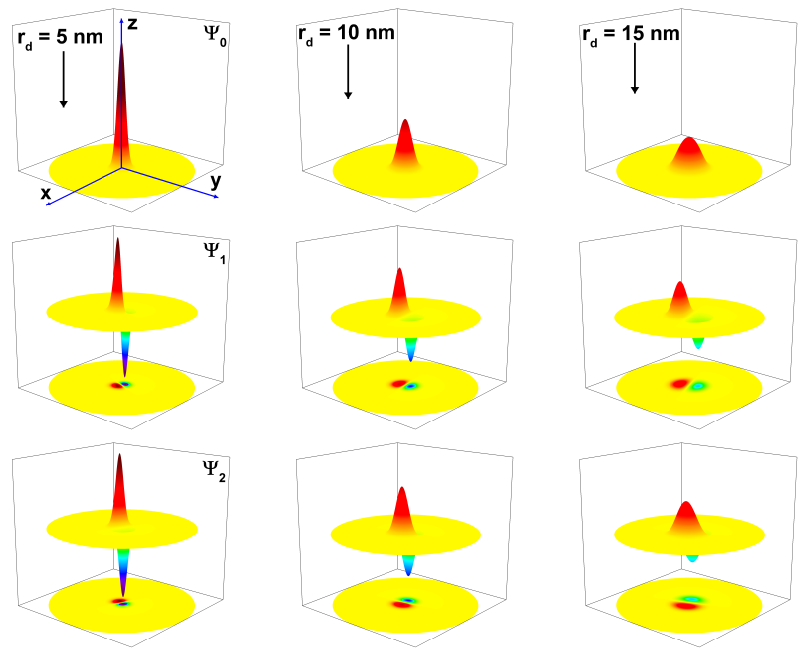

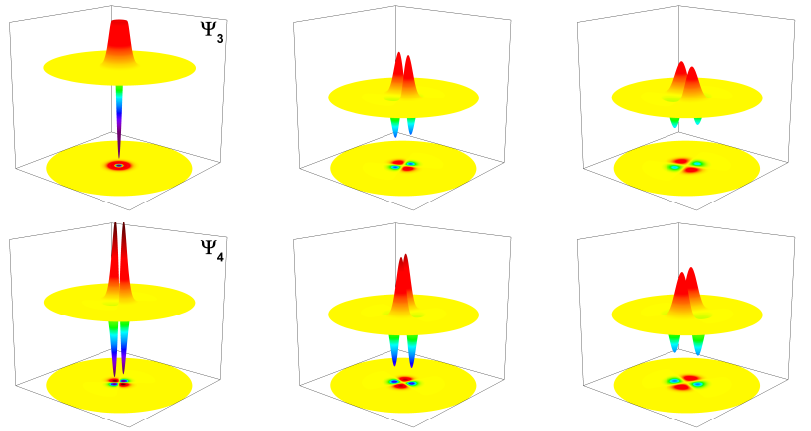

FIG. 3: First five wave functions for a confined electron in GaAs quantum wires. The rows from top to bottom are the ground state and first four excited states, columns from left to right correspond to $r_{d}=5 \mathrm{~nm}, r_{d}=10 \mathrm{~nm}$, and $r_{d}=15 \mathrm{~nm}$, respectively. For all figures the radius of the quantum wire has been set at $R_{0}=50 \mathrm{~nm}, T=10 K$, and $N_{d}=3 \times 10^{19} \mathrm{~cm}^{-3}$.

\section{RESULTS AND DISCUSSION}

\section{A. Results Quantum Well}

In Fig. 4, we present our results for the confining potential, energy levels, Fermi level, and probability densities for the lowest four bound electron states in GaAs Razavy-like QWs, considering four different cases of the external electric and magnetic fields, as well as doping concentration. Despite the inclusion of the third excited state, it does not contribute to the electron density since it is above the Fermi level of the system. From Fig. 4(a), where these three parameters are set to zero, it is observed that the central barrier is high enough such that the ground state is almost-degenerate; corresponding to the ground state - with even symmetry- and the first excited state -with energy very close to the ground state one- showing odd symmetry with respect to the $z=0$ point. The energy difference between the ground state and the second excited state is approximately $150 \mathrm{meV}$. Notice that the second excited state is located above the central barrier. In Fig. 4(b), where an on-center doping concentration of $n_{d}=4.5 \times 10^{19} \mathrm{~cm}^{-3}$ is considered in the absence of external fields (doping of this order is discussed in Ref. [72]), one may observe the appearance of an additional central potential well. The energy minima of the two side QWs, which in Fig. 4(a) have zero value, now show a slight shift towards lower energies. Besides, the minimum of the central well positions itself at an energy slightly higher than the energy of the two lateral minima. 

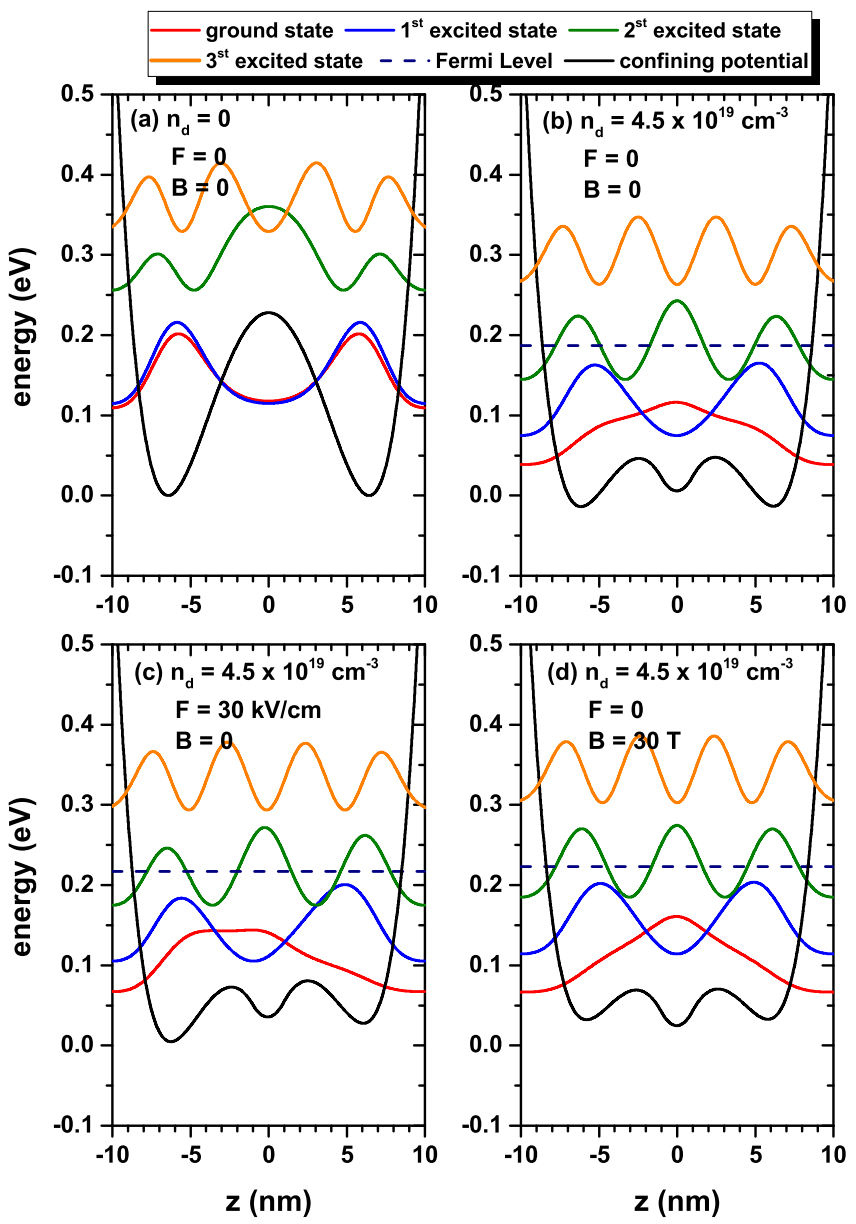

FIG. 4: (color online) Confining potentials, energy levels, Fermi level, and probability density for the lowest four bounded electron states in a GaAs Razavy-like quantum wells. Four different cases of the external fields and doping concentration $\left(F, B, n_{d}\right)$ are considered: $(0,0,0)(\mathrm{a})$, $\left(0,0,4.5 \times 10^{19} \mathrm{~cm}^{-3}\right)(\mathrm{b}),\left(30 \mathrm{kV} / \mathrm{cm}, 0,4.5 \times 10^{19} \mathrm{~cm}^{-3}\right)(\mathrm{c})$, and $\left(0,30 \mathrm{~T}, 4.5 \times 10^{19} \mathrm{~cm}^{-3}\right)(\mathrm{d})$. In (b-d) the Fermi level is depicted with the horizontal line close to $200 \mathrm{meV}$.

Under these conditions, the GaAs Razavy-like double quantum well in the presence of central doping is transformed into a triple quantum well with outer barriers that rapidly diverge, generating an infinite confining potential. The presence of the central well in Fig. 4(b) has the function of coupling the two quantum wells initially observed in Fig. 4(a). In this sense, the breakdown of the ground state degeneration is clearly noticed. It is also observed that the first excited state shows a blue shift of $36 \mathrm{meV}$ concerning the ground state. The latter, whose energy is $3 \mathrm{meV}$ less than the maximum of the finite potential barriers, is an even function with its maximum probability density in the central region; the presence of shoulders associated with the irregularity of the potential well bottom is also well apparent. Besides, the first excited state is an odd function whose maximum probability density is located in the region of the two lateral quantum wells. The second excited state is remarkably insensitive to the shape of the potential well. This is concluded by observing that the two lateral maximums in the probability density present magnitudes slightly lower than that shown by the central maximum. On the other hand, in Fig. 4(c), where a delta-like doping volume density of $n_{d}=4.5 \times 10^{19} \mathrm{~cm}^{-3}$ is combined with an applied electric field of $30 \mathrm{kV} / \mathrm{cm}$ (developments in high electric fields can be seen in Ref. [73, 74]), it is possibel to see that, by breaking the symmetry of the system, the ground state is pushed towards the left-hand side well structure while, due to orthogonality conditions, the first excited state displaces its maximum towards the right-side of the system. The ground state has a quasiconstant probability density in the region $-5 \mathrm{~nm}<z<0$. The comparison between the second excited state in Figs. 4(b) and 4(c) shows a slight blue shift due to the fieldeffect without significant changes in the probability density shape. This effect is associated with the fact that the electric potential is zero at $z=-10 \mathrm{~nm}$. Now, turning to analyzing Fig. 4(d), where a delta-like doping of $n_{d}=4.5 \times 10^{19} \mathrm{~cm}^{-3}$ combines with an applied magnetic field of $30 \mathrm{~T}$ (some experimental and theoretical works in high magnetic fields [75-77]), a parabolic confinement appears associated with the second term in the squared parenthesis of Eq. (5). By comparing the confinement potentials in Figs. 4(b) and 4(d), it is observed that, as a first effect, the parabolic potential is responsible for equalizing in energy the bottom of the three quantum wells. In this way, the central maximum of the ground state probability density is reinforced. Additionally, the three confined states show a blue shift related with the reinforcement of carrier space confinement; noting an increase in the separation in energy between the ground state and the first excited state. The probability density of the second excited state now appears with three maxima, all of them with equal amplitude, reflecting a systematic disappearance of the influence of potential well bottom shape. In Figs. 4(b-d), where central doping is present, it is clearly seen that in the three cases there are only three confined levels with energies lower than the Fermi level.

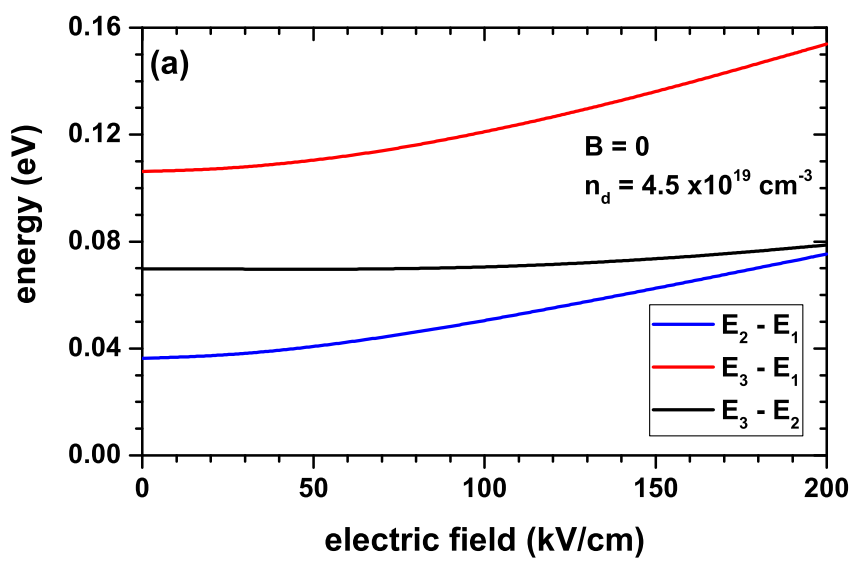




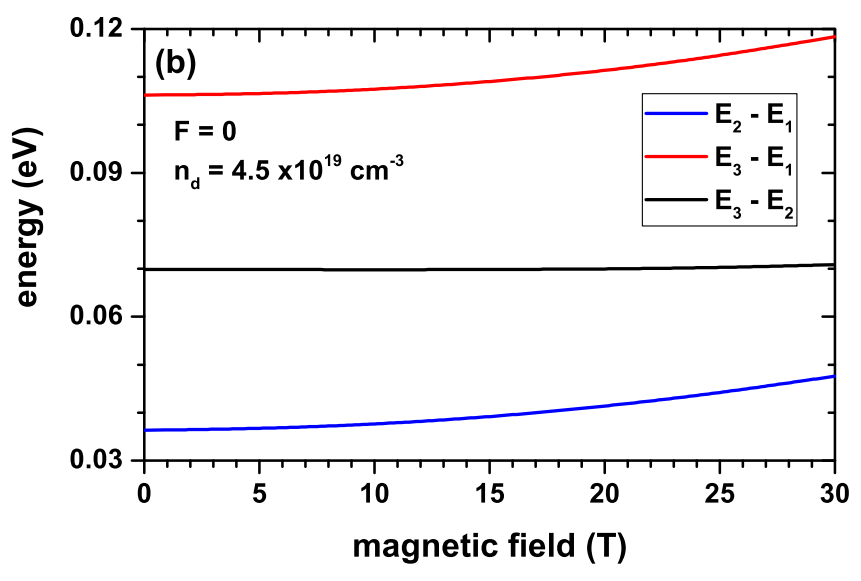

FIG. 5: (color online) Variation of the lowest three energy separations in a central doped GaAs Razavy-like quantum well as a function of the applied electric (a) and magnetic (b) field. Calculations are for $n_{d}=4.5 \times 10^{19} \mathrm{~cm}^{-3}$.

In Fig. 5, we present the results for the variation of the lowest three energy separations $\left(E_{2}-E_{1}, E_{3}-E_{1}\right.$, and $\left.E_{3}-E_{2}\right)$ in a central doped GaAs Razavy-like QW as a function of the applied electric field, without magnetic field effects, Fig. 5(a), and as a function of the applied magnetic field, without electric field effects, Fig. 5(b). The simulations include a fixed delta-like doping concentration, $n_{d}=4.5 \times 10^{19} \mathrm{~cm}^{-3}$. The results in Figs. $5(\mathrm{a})$ and $5(\mathrm{~b})$ are in complete agreement with those presented in Figs. 4(c) and 4(d), respectively. The electric field -variable in Fig. 5(a)- has the function of tilting the potential well profile, generating a greater localization of the carriers towards the $z<0$ region. This effect translates into greater confinement of the carriers, thereby increasing the separation between the confined levels and consequently the transition energies. The quasi-parallel behavior of the $E_{2}-E_{1}$ and $E_{3}-E_{1}$ curves justifies the quasi-constant behavior with the electric field of the $E_{3}-E_{2}$ curve which, in the whole range of electric fields considered, only presents a variation of $8 \mathrm{meV}$; less than $10 \%$. It is important to note that the wave functions associated to the three states in Fig. 5(a) lose their odd or even symmetry with respect to the $z=0$ point, which means that all inter-subband transitions are allowed. As commented, the magnetic field -the variable in Fig. 5(b)is responsible for a parabolic potential whose effect is to increase the localization of all confined states within the region close to $z=0$. This greater localization of the states translates into an increase in the transition energies with the applied magnetic field. Due to its greater extension in space, the second excited state $\left(\Phi_{3}\right)$ is the one most susceptible to being spatially modified by the effect of the applied magnetic field. The ground state $\left(\Phi_{1}\right)$, which has its maximum probability density close to the $z=0$ region, is essentially insensitive to the effects of the magnetic field. Combining these two aspects, one may understand why, as in Fig. 5(a), the $E_{2}-E_{1}$ and $E_{3}-E_{1}$ transition energies are the most sensitive to the magnetic field, with a growing behavior and parallel to each other. This justifies that in the range of magnetic fields, the $E_{3}-E_{2}$ transition energy is constant. The wave functions associated with the three states considered in the transitions of Fig. 5(b) preserve their even or odd symmetries. The $\Phi_{1}$ and $\Phi_{3}$ states are even functions concerning $z=0$ while the $\Phi_{1}$ state is an odd function. Consequently, only the $1 \rightarrow 2$ and $2 \rightarrow 3$ transitions are allowed whereas the $1 \rightarrow 3$ transition is forbidden.

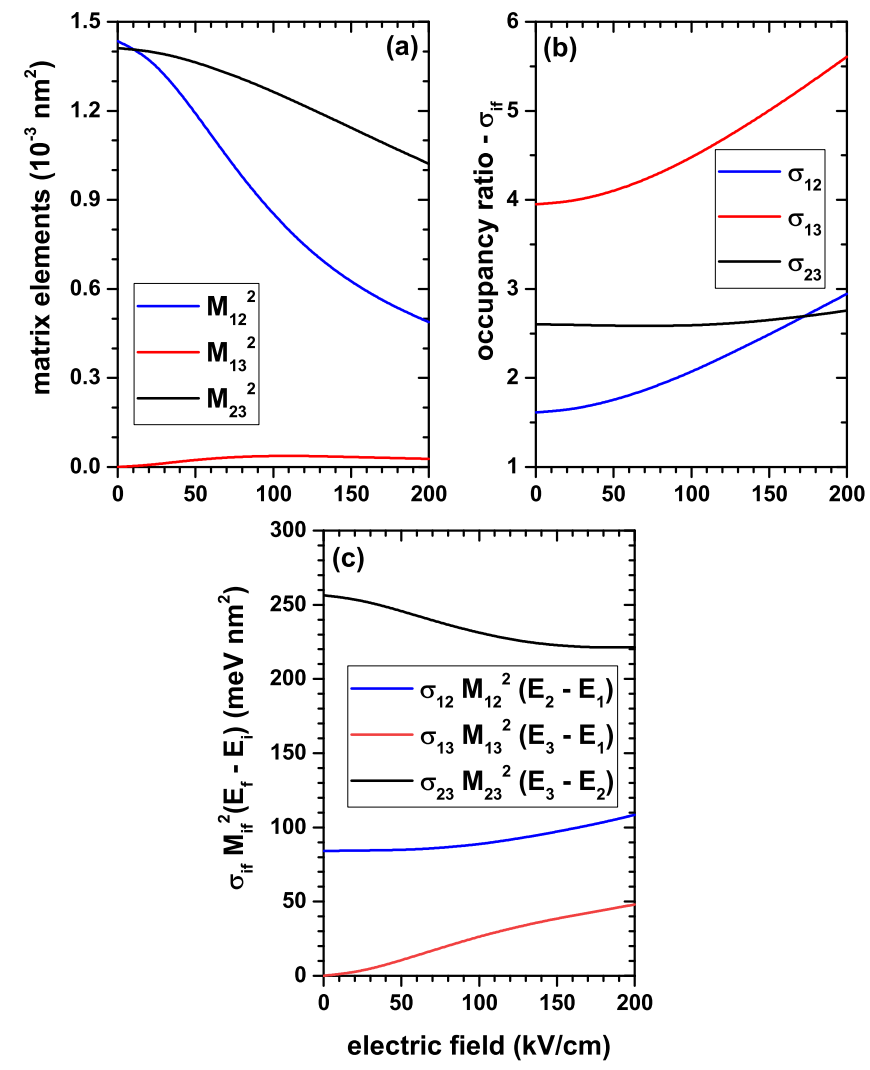

FIG. 6: (color online) Variation of the reduced dipole matrix elements- $\left|M_{i f}\right|^{2}(\mathrm{a})$, the occupancy ratio- $\sigma_{i f}(\mathrm{~b})$, and the function $F_{i f}=\sigma_{i f}\left|M_{i f}\right|^{2}\left(E_{f}-E_{i}\right)$ (c) in a central doped GaAs Razavy-like quantum well as a function of the applied electric field, for zero magnetic field. The results are for $n_{d}=4.5 \times 10^{19} \mathrm{~cm}^{-3}$. 


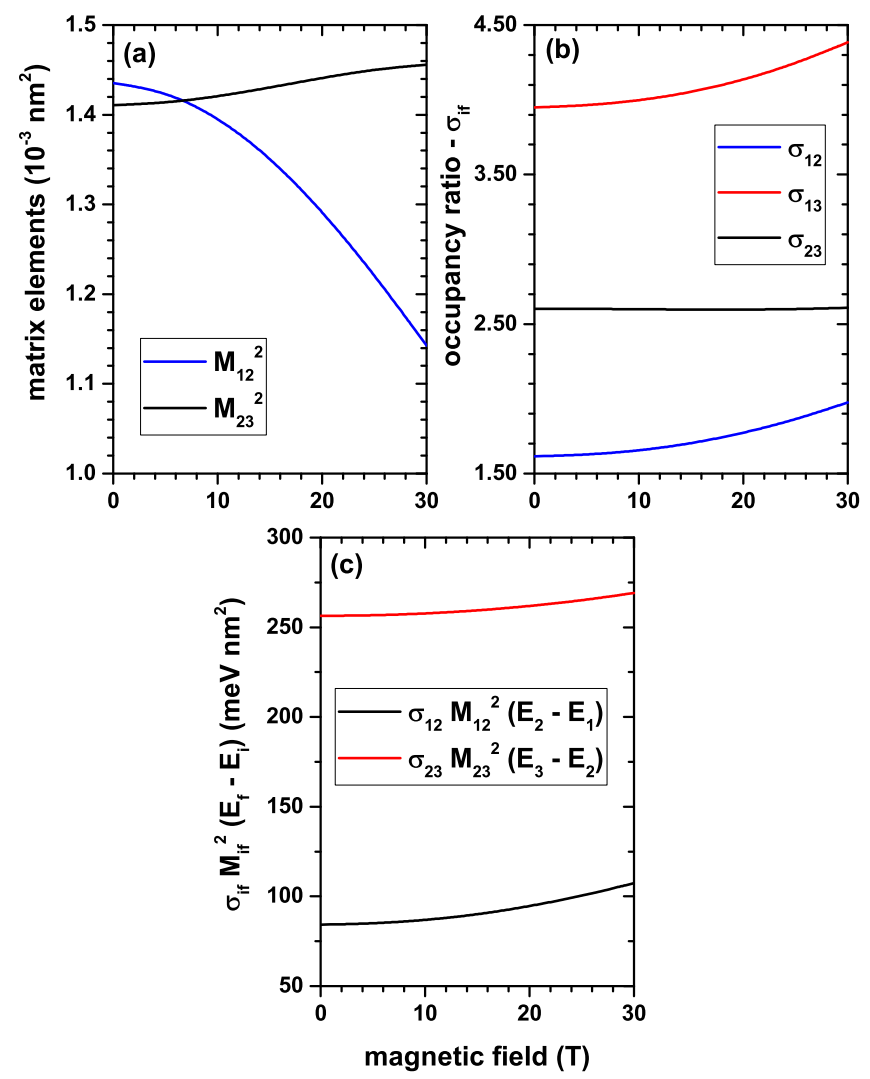

FIG. 7: (color online) Variation of the reduced dipole matrix elements- $\left|M_{i f}\right|^{2}(\mathrm{a})$, the occupancy ratio- $\sigma_{i f}(\mathrm{~b})$, and the function $F_{i f}=\sigma_{i f}\left|M_{i f}\right|^{2}\left(E_{f}-E_{i}\right)$ (c) in a central doped GaAs Razavy-like quantum well as a function of the applied magnetic field for zero electric field. The results are for $n_{d}=4.5 \times 10^{19} \mathrm{~cm}^{-3}$.

In Figs. 6 and 7 we present the variation of the reduced dipole matrix elements- $\left|M_{i f}\right|^{2}$ (a), the occupancy ratio- $\sigma_{i f}(\mathrm{~b})$, and the function $F_{i f}=\sigma_{i f}\left|M_{i f}\right|^{2}\left(E_{f}-E_{i}\right)$ (c) in a central doped GaAs Razavy-like QW, plotted as functions of the applied electric field, for zero magnetic field; and as functions of the applied magnetic field, for zero electric field, respectively. The results correspond to a delta-like doping with $n_{d}=4.5 \times 10^{19} \mathrm{~cm}^{-3}$. As already said before, since the presence of an electric field removes the even or odd symmetry of the confined states then all the transitions between lowest three confined states are allowed, as shown in Fig. 6(a). therefore all corresponding intersubband $\left|M_{i f}\right|^{2}$ elements are nonzero. However, as seen from Fig. 7(a), since the ground and the second excited states are even functions, then $M_{13}^{2}=0$.

Taking into account that both the electric and magnetic fields have the effect of increasing the location of carriers, then the spatial extension where the wave functions spread out decreases. This explains the decreasing tendency of the reduced dipole matrix elements in Figs. $6(\mathrm{a})$ and $7(\mathrm{a})$ as functions of the electric and magnetic fields, respectively. An unexpected situation occurs with the term $M_{23}^{2}$ in Fig. 7(a). Its growing character with the magnetic field is essentially associated with the in- creasing spatial overlap of $\Phi_{2}$ and $\Phi_{3}$ states. Note that the magnetic field is responsible for the increment (decrement) of the probability density of $\Phi_{3}$ state in the region where $\Phi_{2}$ has its two maxima values (has its minimum value). From Fig. 6(a) it is also possible to observe that the term $M_{13}^{2}$ is approximately zero over the entire range of electric fields. Despite the symmetry breaking in the wave functions, the Fig. 4(a) shows that the electric field shifts the only maximum present in the $\Phi_{1}$ state towards the region where $\Phi_{3}$ has a minimum. For this reason, the overlap between these two wave functions tends to zero, giving an approximately negligible value of the dipole matrix element. From the increasing behavior of $\sigma_{12}$ and $\sigma_{13}$ and approximately constant of $\sigma_{23}$ in Figs. 6(b) and $7(\mathrm{~b})$, it is concluded that in general the energy separation between the $\Phi_{2}$ and $\Phi_{3}$ states is approximately constant with the electric and magnetic fields and that the transition energy between $\Phi_{1}$ and $\Phi_{2}$ (or between $\Phi_{1}$ and $\left.\Phi_{3}\right)$ is an increasing function of the electric and magnetic fields. As the strength of the two external probe fields augments, an increase in confinement is observed, greater spacing between levels appears; and it is much more difficult to thermally excite the electrons from the ground to excited states. This explains why there is an increase in the occupancy rate with the electric and magnetic field. Figs. 6(c) and 7(c), where we present the function $F_{i f}=\sigma_{i f}\left|M_{i f}\right|^{2}\left(E_{f}-E_{i}\right)$, show quite intricate mixing behavior of the three factors involved, which can be summarized as follows. For the electric field effects in Fig. 6(c), it is observed that: i) $F_{12}$ and $F_{13}$ are dominated by the behavior of their corresponding $\sigma_{i f}$ and $E_{f}-E_{i}$ parameters and ii) $F_{23}$ is dominated by the behavior of $M_{23}^{2}$. In the case of Fig. $7(\mathrm{c})$, the nonzero $F_{12}$ and $F_{23}$ functions increase with the magnetic field and essentially follow the transition energy behavior.

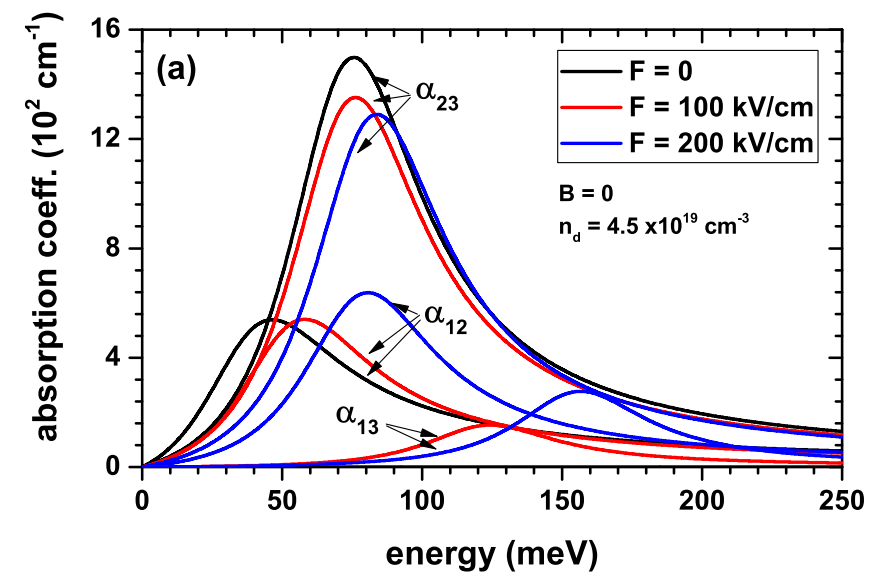




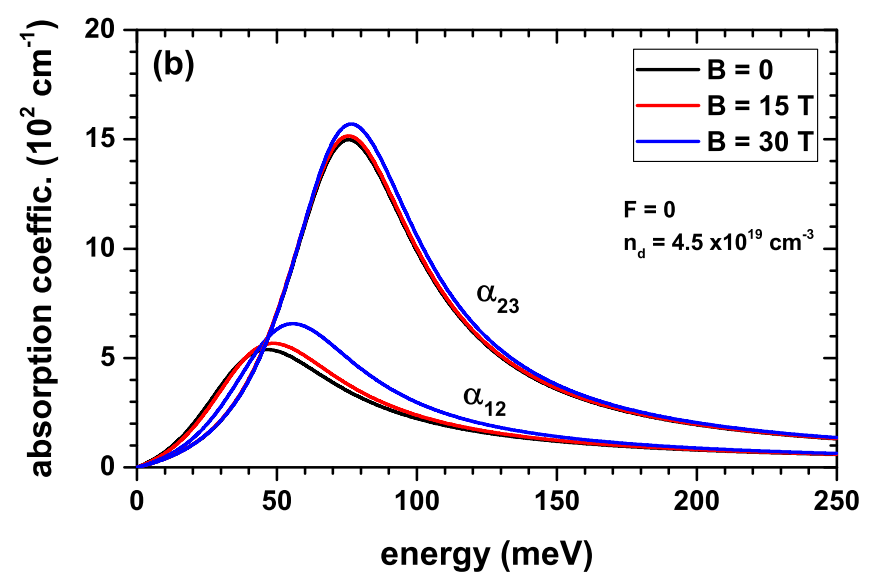

FIG. 8: (color online) Variation of the total optical absorption coefficient in a central doped GaAs Razavy-like quantum well as a function of the $z$-polarized incident photon energy for three different values of the applied electric field, for zero magnetic field (a) and for three values of the applied magnetic, for zero electric field (b). The results are for $n_{d}=4.5 \times$ $10^{19} \mathrm{~cm}^{-3}$.

In Fig. 8 we present the variation of the total optical absorption coefficient in a central $\delta$-like doped GaAs Razavy-like QW as a function of the $z$-polarized incident photon energy for three different values of the applied electric field, with $B=0$ (a) and for three values of the applied magnetic, with $F=0$ (b) and keeping constant the $n_{d}$ concentration. According to Eq. (11), the magnitude of the first-order correction resonant peak of the optical absorption coefficient is proportional to $F_{i f}=\mid M_{i f}^{2} \sigma_{i f}\left(E_{f}-E_{i}\right)$ which is precisely the quantity reported in Figs. 6(c) and 6(c). The incident radiation intensity chosen in this study is $I=0.5 \mathrm{MW} / \mathrm{cm}^{2}$ and, under such assumption, a clear dominance of the linear contribution to the total light absorption is present. Note that the magnitude of the maxima of $\alpha_{i j}$ in all the curves of Fig. 8 follow the behavior of $F_{i j}$ reported in Figs. 6 and 7. For example, in Fig. 6(c), we can see that $F_{23}$ decreases with the electric field, a situation that is identical to the decrease of the resonant peak of $\alpha_{23}$ in the three corresponding curves in Fig. 8(a) as it increases the electric field. The almost constant behavior of the magnitude of the resonant peak of $\alpha_{23}$ in Fig. 8(b) is directly related to the slight variation shown by $F_{23}$ in Fig. $7(\mathrm{c})$. The absence of the $\alpha_{13}$ coefficient in Fig. 8(b) for all the magnetic fields strengths considered and in Fig. 8(a) for $F=0$ is since the $\alpha_{13}$ transitions are forbidden between states that have the same even symmetry. As mentioned, in general, the electric and magnetic fields used as external probes in this study are responsible for the increase in carriers' confinement, which finally translates into a greater spacing between adjacent levels and consequently in an increase in the transition energies. This fact is in perfect coherence with the blue shift shown by the resonant peaks of the total absorption coefficient as the electric field increases, as shown in Fig. 8(a), or as the magnetic field increases, as shown in Fig. 8(b).
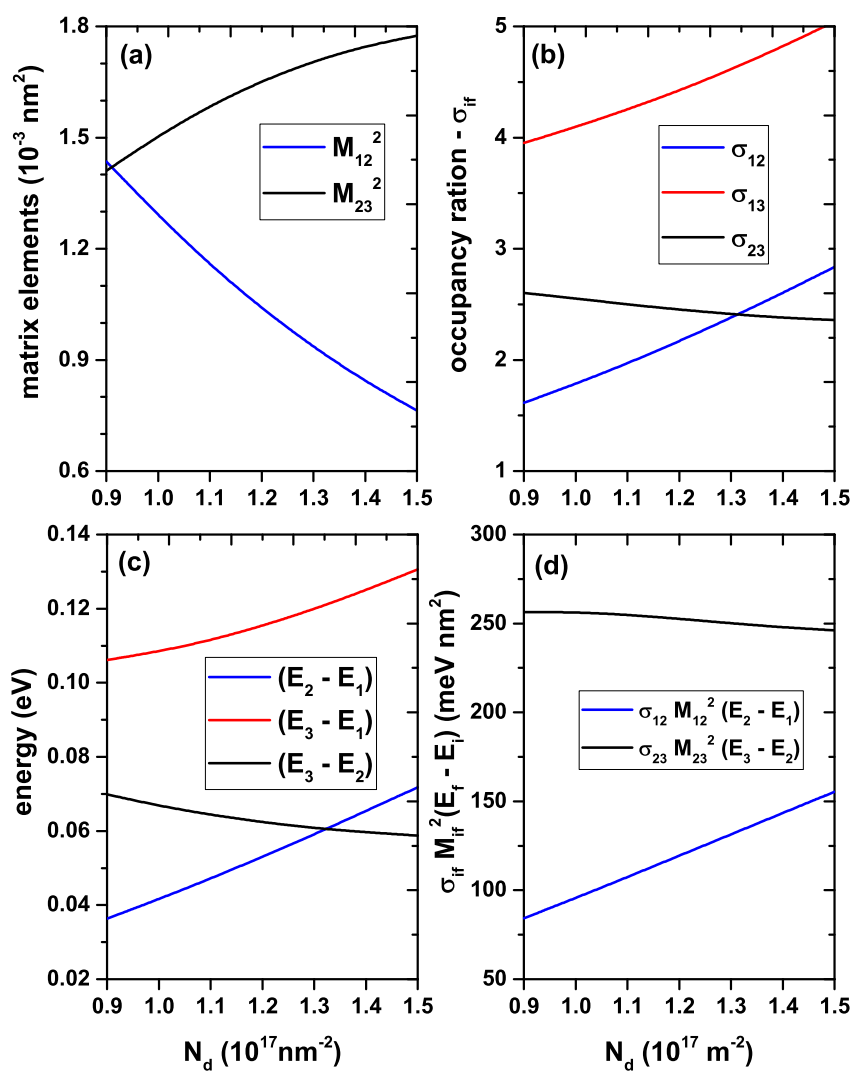

FIG. 9: (color online) Variation of the reduced dipole matrix elements- $\left|M_{i f}\right|^{2}$ (a), the occupancy ratio- $\sigma_{i f}(\mathrm{~b})$, the transition energies- $E f-E_{i}$ (c), and the function $F_{i f}=$ $\sigma_{i f}\left|M_{i f}\right|^{2}\left(E_{f}-E_{i}\right)(\mathrm{d})$ in a central doped GaAs Razavy-like quantum well as a function of the $n_{d}$-doping concentration. The results are for $F=0$ and $B=0$.

In Fig. 9 we present the variation of the reduced dipole matrix elements (a), the occupancy ratio (b), the transition energies (c), and the $F_{i f}$-function (d) in a central $\delta$-like doped GaAs Razavy-like QW as a function of the doping volume concentration for zero electric and magnetic field. As shown in Fig. 4(b), central doping creates a potential well in the center of the structure, giving rise to a system of three coupled wells in the Razavylike double quantum well system studied here. When $n_{d}=4.5 \times 10^{19} \mathrm{~cm}^{-3}$, see Fig. $4(\mathrm{~b})$, it is observed that the central well, where doping exists, has its minimum with energy slightly higher than the original two minimums of the Razavy-like double quantum well. As $n_{d}$ grows from that value, the two potential barriers that separate the wells decrease in their heights until they finally collapse, and the system evolves from three potential wells to a single QW that drags the ground state successively towards lower energies. This effect is responsible for the increasing behavior of the $E_{2}-E_{1}$ and $E_{3}-E_{1}$ transition energies in Fig. 9(c). The central potential well, with increasing $n_{d}$, confines within it the $\Phi_{1}$ and $\Phi_{2}$ states, with which an increasing behavior appears reinforced by the 
confinement of the $E_{2}-E_{1}$ transition energy. This fact justifies that the slope with $n_{d}$ of the $E_{2}-E_{1}$ transition is higher than that exhibited by $E_{3}-E_{1}$, which explains the decreasing character of the $E_{3}-E_{2}$ transition. As the $\Phi_{1}$ and $\Phi_{2}$ states are confined in the central well, a greater localization oaround $z=0$ gives rise to the diminishing character of $M_{12}^{2}$ in Fig. 9(a). Due to the increment in the localization of $\Phi_{2}$ close to $z=0$ and that the spatial extension of $\Phi_{3}$ shows negligible changes with the increase of $n_{d}$, the augmenting character of $M_{23}^{2}$ is justified. The variations of $\sigma_{i f}$ in Fig. 9(b) exactly follow the behavior of $E_{f}-E_{i}$ as previously justified. Fig. 9(d) shows unequivocally that in the $F_{i f}$ function, the dominant factors are the transition energies and the occupancy ratio.

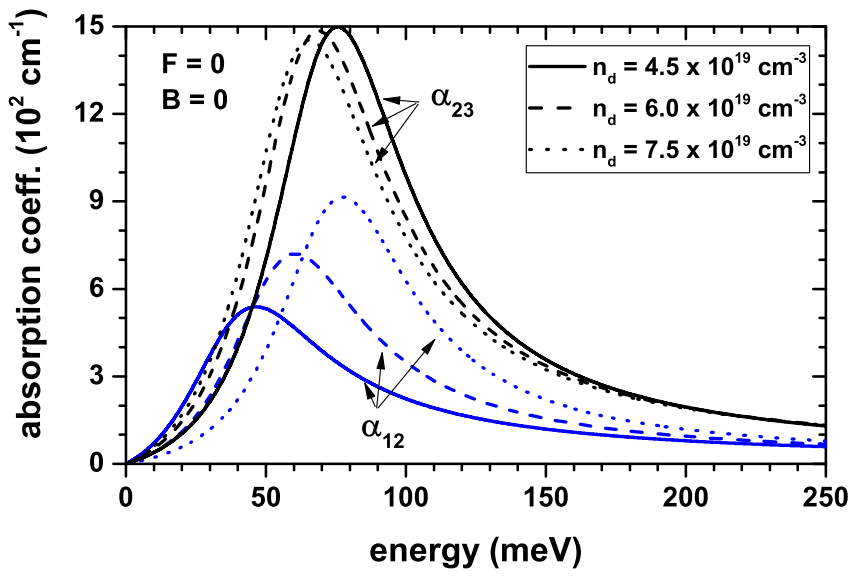

FIG. 10: (color online) Variation of the total optical absorption coefficient $\alpha_{12}$ and $\alpha_{23}$ in a central doped GaAs Razavylike quantum well as a function of the incident photon energy for three different values of the $n_{d}$-doping concentration. The results are for $F=0$ and $B=0$.

Figure 10 contains our results for the variation of the total optical absorption coefficient $\alpha_{12}$ and $\alpha_{23}$ in the investigated GaAs Razavy-like QW with on-center deltalike doping, plotted as a function of the incident photon energy for three different values of the $n_{d}$-doping concentration in the absence of any external field. The absence of the $\alpha_{13}$ transitions is justified by the symmetric nature of the structure, in which case both the ground state and the second excited state are even functions between which the dipole matrix element is zero. The red/blue shift of $\alpha_{12} / \alpha_{23}$ is explained by the results in Fig. 9(c). Likewise, Fig. 9(d) justifies the variations in the magnitudes of the resonant structures and again makes it possible to argue that the first order correction is the dominant one in the optical absorption coefficient.

\section{B. Results Quantum Wire}

In this subsection, we present the results of calculations for the two-dimensional QWR system with a Razavy potential as described in section 2. As in the previous case, the following parameters have been set: effective mass of electron in GaAs $m^{*}=0.067 m_{0}$, where $m_{0}$ is the mass of the free electron and dielectric constant $\epsilon_{r}=12.9$. Coupled differential equations have been solved by means of the finite element method with the following setup: inner mesh with triangular shaped elements, number of elements 6550, edge elements 160, mesh vertices 3356, maximum number of iterations for Self-consistent method 40, absolute tolerance for Self-consistent method $10^{-6}$.
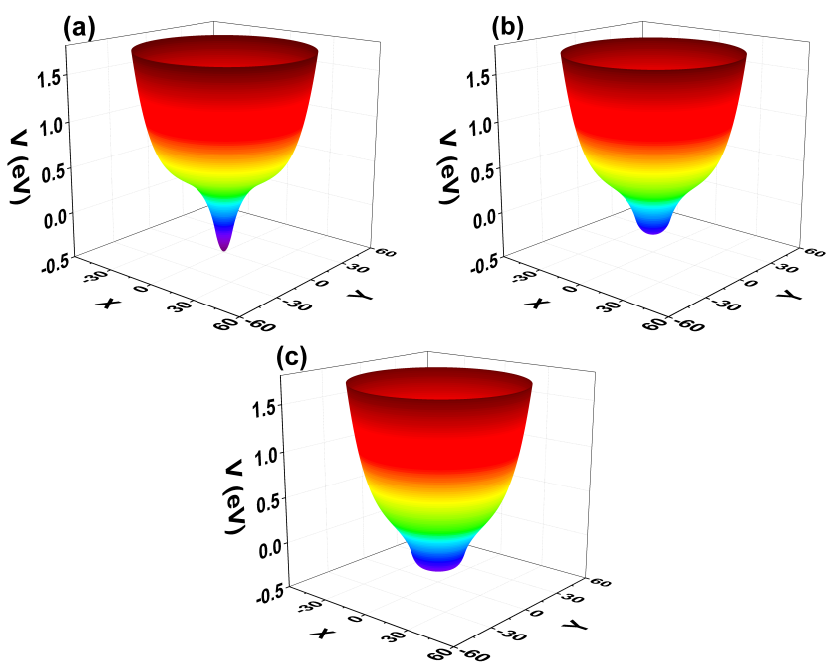

FIG. 11: (color online) Variation of the total optical absorption coefficient $\alpha_{12}$ and $\alpha_{23}$ in a central doped GaAs Razavylike quantum well as a function of the incident photon energy for three different values of the $n_{d}$-doping concentration. The results are for $F=0$ and $B=0$.

Figure 11 shows the self-consistently determined confinement potential for three different radii, $r_{d}$, for the GaAs QWR: $r_{d}=5 \mathrm{~nm}$ (a), $r_{d}=10 \mathrm{~nm}$ (b), and $r_{d}=15 \mathrm{~nm}$ (c). Calculations are with $R_{0}=50 \mathrm{~nm}$, $T=10 \mathrm{~K}$, and $N_{d}=3 \times 10^{19} \mathrm{~cm}^{-3}$. The red color in each figure indicates larger values for the potential, while the blue color indicates the smaller values for it. For the three cases studied, the self-consistent potential presents a stable equilibrium point at $x=0$ (center of symmetry of the system), which corresponds to a minimum potential and therefore, the electrons will be practically confined around the central region of the QW, feeling an infinite potential at the system boundary. For $r_{d}=5 \mathrm{~nm}$, the potential presents a very sharp peak near the center of the structure generated by the rearrangement of charges. Such a charge distribution practically resembles a delta type doping in the system, given the very small value of $r_{d}$ compared to the total radius of the wire $R_{0}$. For the larger radii of the on-center doping region the sharp peak no longer appears. Instead, the profile shows a flatter bottom around the center of symmetry. However, in all cases, these lower structures correspond to smaller values of the potential energy, compared to the entire cross section of the QW. This indicates that electrons will tend to localize towards the interior this central region. 

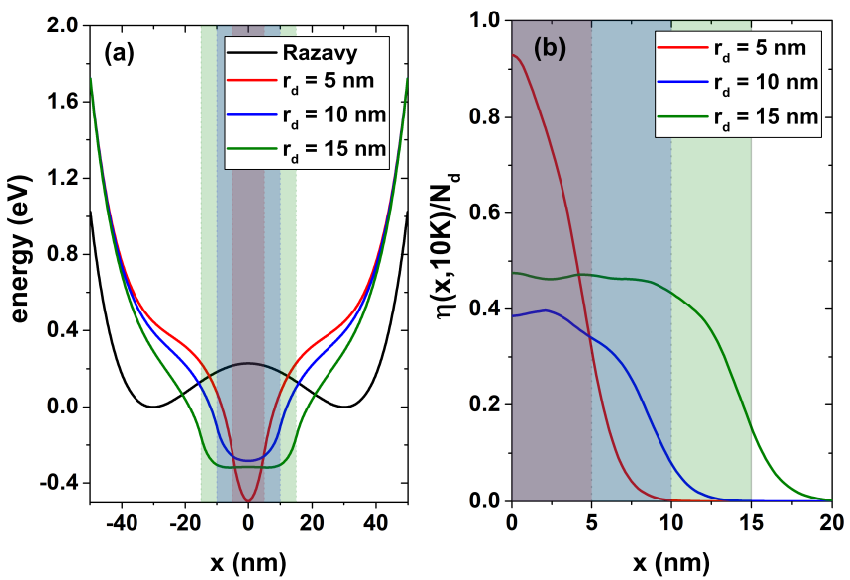

FIG. 12: Projection in the $x$ direction of the self-consistent potential for the three doping radio $r_{d}$ studied, 5, 10 and $15 \mathrm{~nm}$. The black curve corresponds to the Razavy potential inside QW, included as a reference (a). Self-consistent electron densities corresponding to each of the potentials presented in (a) maintaining the same projection direction in the $x$ direction (b). Calculations are with $R_{0}=50 \mathrm{~nm}, T=10 \mathrm{~K}$, and $N_{d}=3 \times 10^{19} \mathrm{~cm}^{-3}$.

In the Fig. 12(a) we plot the projection along the $x$ direction of the self-consistent potential for the three values of doping radii $r_{d}$ considered: 5,10 and $15 \mathrm{~nm}$. The black curve corresponds to the Razavy potential inside QWR, which is included as a reference. Calculations consider $R_{0}=50 \mathrm{~nm}, T=10 \mathrm{~K}$, and $N_{d}=3 \times 10^{19} \mathrm{~cm}^{-3}$. Vertical bars indicate the different doping radii, $r_{d}$. The potential reaches a minimum value of $-0.49 \mathrm{eV}$, for $r_{d}=5 \mathrm{~nm}$, followed that with $r_{d}=15 \mathrm{~nm}$, where the minimum is at $-0.32 \mathrm{eV}$ and, finally, when $r_{d}=10 \mathrm{~nm}$ the minimum is $-0.28 \mathrm{eV}$. The value of these minima is crucial for the positioning of the electronic states as well as to quantify their contribution to the electron density in the system. In the regions close to the QWR boundary, all potentials converge to a single value of $1.72 \mathrm{eV}$ which corresponds to the sum of the potential due to the Fermi level Pinning in GaAs which is $0.7 \mathrm{eV}$ plus the Razavy potential at the boundary that is equal to $1.02 \mathrm{eV}$. That is, the potential at the boundary is not modified by the effect of the doped delta layer in the central region of the wire. Note the similarity of the black curve that corresponds to the projection of the Razavy potential on the $x$-axis with the potential presented in Figure 4(a) (blue curve) for the one-dimensional QW.

Figure 12(b) shows the electron density for each configuration of the system. Due to the symmetry of these quantities, they have are plotted only from the center of the structure towards the border. The colors on each curve exactly match to each of the potentials in Fig. 12(a). The electron density that reaches the maximum value is that corresponding to $r_{d}=5 \mathrm{~nm}$, which is, precisely, the cylindrical delta-doping region with the smallest radius. In this case, electrons are confined within a circular cross-section that does not exceed $20 \mathrm{~nm}$ in diam- eter, and the behavior of electron density is completely decreasing as we move away from the center of symmetry of the system. For the radii $r_{d}=10 \mathrm{~nm}$ and $r_{d}=15 \mathrm{~nm}$, the densities decrease in magnitude, having a distribution along the cross section of the wire with diameters not greater than $30 \mathrm{~nm}$ and $40 \mathrm{~nm}$ respectively. The system that reaches the lowest magnitude in electron density is the system with $r_{d}=10 \mathrm{~nm}$, which, as described before, corresponds to the highest potential in the center of the system. It should be noted that density profiles generated for $r_{d}=10 \mathrm{~nm}$ and $15 \mathrm{~nm}$ are not always totally decreasing, but exhibit the so-called Friedel-like oscillations, which appear as irregularities in the electron density near the center of symmetry of the system.

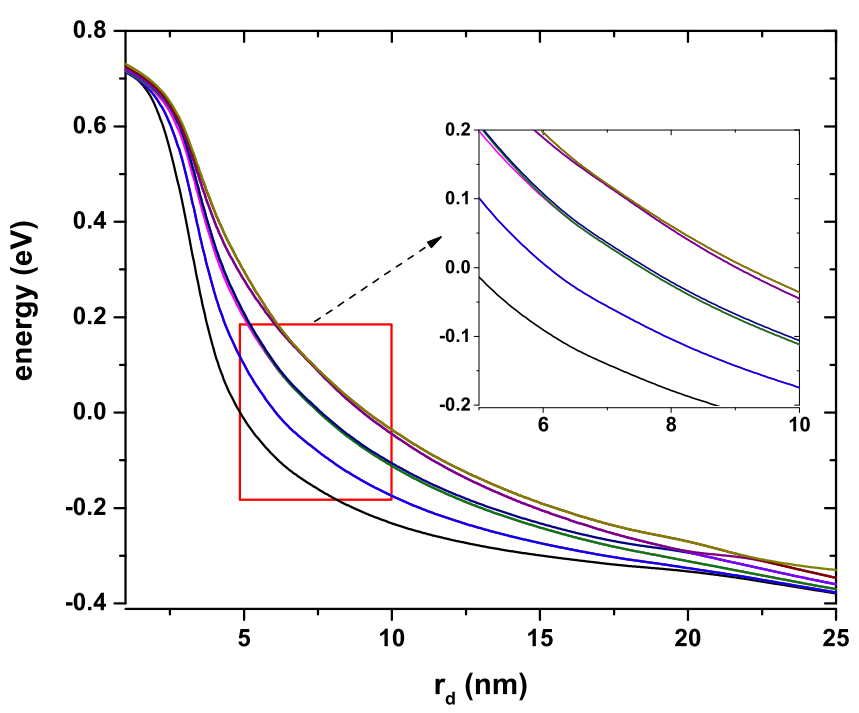

FIG. 13: First energy levels for a confined electron in a GaAs quantum wire as a function of the $r_{d}$ parameter. The inset shows a zoom for $r_{d}$ values between $5 \mathrm{~nm}$ and $10 \mathrm{~nm}$. Calculations are with $R_{0}=50 \mathrm{~nm}, T=10 \mathrm{~K}$, and $N_{d}=$ $3 \times 10^{19} \mathrm{~cm}^{-3}$.

Figure 13 shows the first energy levels for a confined electron in a GaAs QWR as functions of the $r_{d}$ parameter. The inset shows a zoom for $r_{d}$ values between $5 \mathrm{~nm}$ and $10 \mathrm{~nm}$. Calculations correspond to $R_{0}=50 \mathrm{~nm}$, $T=10 \mathrm{~K}$, and $N_{d}=3 \times 10^{19} \mathrm{~cm}^{-3}$. For all states there is a clear monotonous decreasing behavior. For $r_{d}$ values smaller than $2.5 \mathrm{~nm}$, the states presented are very close to each other around an average value of $0.72 \mathrm{eV}$. For $r_{d}$ values bigger than $2.5 \mathrm{~nm}$, the states present a considerable separation and there are crossovers between some excited states. The inset shows the region $5 \mathrm{~nm}$ $<r_{d}<10 \mathrm{~nm}$ in which it is possible to clearly observe some of these crossovers between states, with double or even triple degenerations occurring at specific points. For $r_{d}$ values greater than $20 \mathrm{~nm}$, the states again tend to be closer together due to the distribution of charges along the cross section of the wire. 


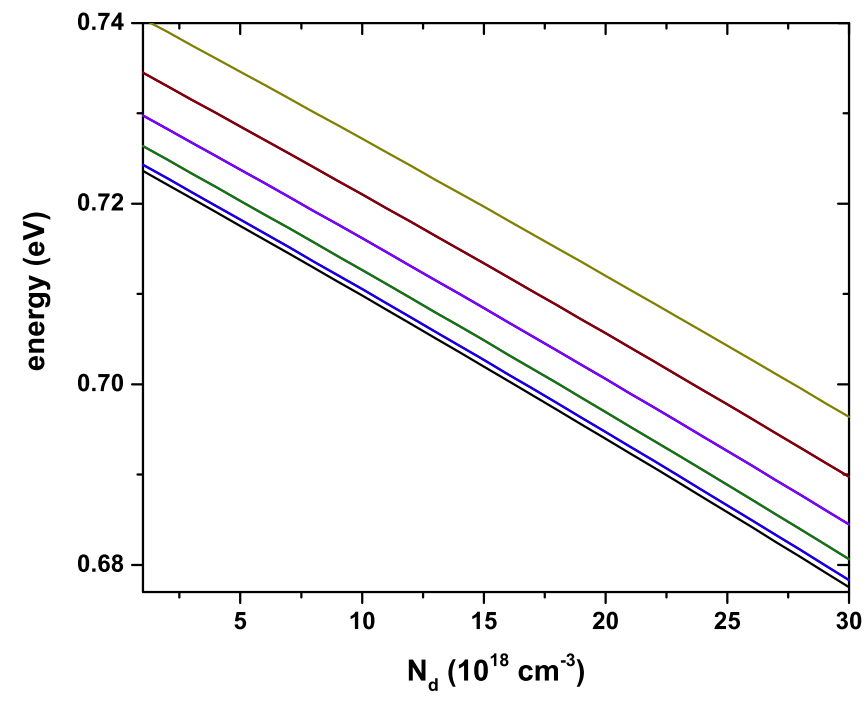

FIG. 14: First energy levels for a confined electron in a GaAs quantum wire as a function of the $N_{d}$ parameter. Calculations are with $R_{0}=50 \mathrm{~nm}, r_{d}=2 \mathrm{~nm}$, and $T=10 \mathrm{~K}$.

The plot in Figure 14 shows the lowest energy levels for a confined electron in a GaAs QWR as a function of the $N_{d}$ parameter. Results presented are for $R_{0}=50 \mathrm{~nm}$, $r_{d}=2 \mathrm{~nm}$, and $T=10 \mathrm{~K}$. With this setup, all states present a parallel and approximately linear behavior, existing degeneration of order two for all the states except for the ground one. It should be noted that, unlike Fig. 13 in which the $r_{d}$ is varied, there are no longer any crossovers between the states. Note that the first excited state and the ground state present very close energies, with a separation of approximately $0.7 \mathrm{meV}$, whilst the other excited states exhibit a more notable separation.
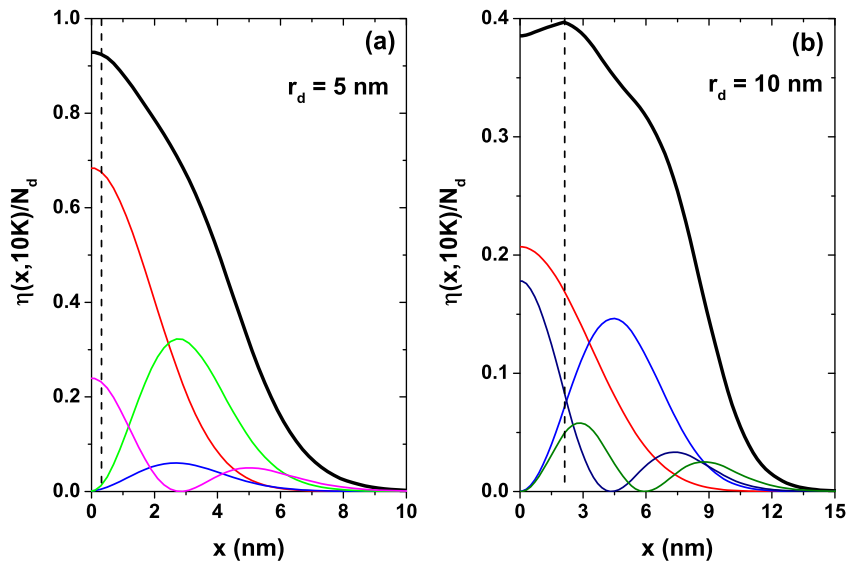

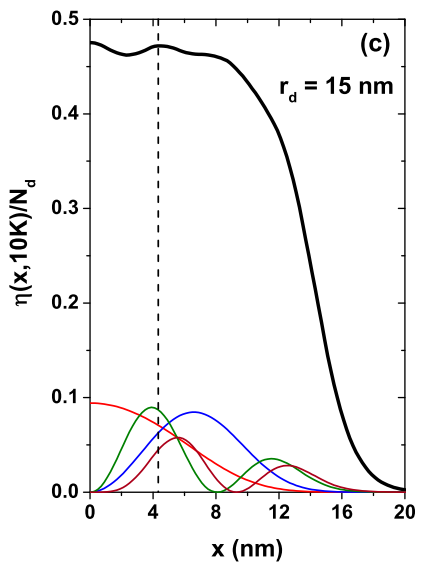

FIG. 15: Electron density and $\left|\psi_{i}(x, y=0)\right|^{2}$ that contribute with the highest percentage to the density profile at the points marked with the dashed lines. Results are for $r_{d}=5 \mathrm{~nm}(\mathbf{a})$, $r_{d}=10 \mathrm{~nm}(\mathbf{b})$, and $r_{d}=15 \mathrm{~nm}$ (c) QW. Calculations are with $R_{0}=50 \mathrm{~nm}, T=10 \mathrm{~K}$, and $N_{d}=3 \times 10^{19} \mathrm{~cm}^{-3}$.

The electron density appears in Fig. 15 as a function of the distance from the QWR center (along the $x$ direction), together with the those quantities, $\left|\psi_{i}(x, y=0)\right|^{2}$, that contribute with the highest percentage to the density profile at the points marked with the dashed lines. Results correspond for $r_{d}=5 \mathrm{~nm}(\mathrm{a}), r_{d}=10 \mathrm{~nm}(\mathrm{~b})$, and $r_{d}=15 \mathrm{~nm}$ (c) QWR. Calculations were performed with $R_{0}=50 \mathrm{~nm}, T=10 \mathrm{~K}$, and $N_{d}=3 \times 10^{19} \mathrm{~cm}^{-3}$.

In Fig. 15(a) the projection of the electron density along the $x$ direction is presented for $r_{d}=5 \mathrm{~nm}$ and, for this configuration, there are no oscillations in the density profile. The dashed line corresponds to the point at which the percentage contribution of each of the states of the system to the total electron density has been calculated. This contribution is presented in detail in Table 1. For this first system, the electron density profile is due only to the contribution of four states. the highest contribution is due to the ground state $\psi_{0}$ with $73.1 \%$ corresponding to the red curve in Fig. 15(a), followed by state $\psi_{3}$ with $25.1 \%$. No Friedel-like oscillations are present in this case. Fig. 15(b) contains the electron density profile for $r_{d}=10 \mathrm{~nm}$ and the states that present a contribution greater than $10 \%$ at the point $x=2.1 \mathrm{~nm}$ marked with the dashed line. For the total electron density, a contribution of eight states is present as reported in Table 1. Again, the state that has a greater contribution is $\psi_{0}$, with $42.8 \%$, and corresponds to the red curve in the figure. It is followed by the state $\psi_{5}$ which is already a higher state with $21.2 \%$ and later the state $\psi_{1}$ with $17.8 \%$. The appearance of this oscillation is mainly due to the occupation of the lowest states in the system $\left(\psi_{0}\right.$ and $\left.\psi_{1}\right)$ with a contribution greater than $60 \%$. Fig. 15(c) presents the electron density profile for $r_{d}=15 \mathrm{~nm}$, together with the probability densities of states that present a contribution greater than $9 \%$ to the electron density at the point $x=4.3 \mathrm{~nm}$-marked with the dashed line. Comparing with figures (a) and (b), in this case the first oscillation appears further from the 
center of symmetry of the system. It should be noted that the electron density for this case contains contributions from thirty states, however, none of them exceeds the $19 \%$ contribution to this Friedel-like oscillation as evidenced in Table 1 . The state that contributes the most (state with the highest occupancy at this specific point) is one of the higher states, $\psi_{8}$, with $18.5 \%$ followed by the ground state, $\psi_{0}$, with $15.1 \%$. The Table 1 does not include states with a contribution lower than $1 \%$. Comparing the three values of $r_{d}$ and the data collected in Table 1, it is possible to conclude that as the value of $r_{d}$ augments, the ground state ceases to be the predominant state in terms of occupancy. Instead, a significant contribution from the highest states in the system will occur.

\begin{tabular}{|c|l|l|l|}
\hline & \multicolumn{3}{|l|}{ Contribution by state $(\%)$} \\
\hline$r_{d}(\mathrm{~nm}) \Rightarrow$ & 5 & 10 & 15 \\
\hline$\psi_{0}$ & 73.1 & 42.8 & 15.1 \\
$\psi_{1}$ & 0.5 & 17.8 & 13.2 \\
$\psi_{2}$ & 1.3 & 0.3 & 1.7 \\
$\psi_{3}$ & 25.1 & 1.0 & 1.6 \\
$\psi_{4}$ & & 0.9 & 3.0 \\
$\psi_{5}$ & & 21.2 & 6.0 \\
$\psi_{8}$ & & 12.3 & 18.5 \\
$\psi_{9}$ & & 3.7 & 4.2 \\
$\psi_{12}$ & & & 9.4 \\
$\psi_{13}$ & & & 2.7 \\
$\psi_{18}$ & & & 3.3 \\
$\psi_{19}$ & & & 7.9 \\
$\psi_{20}$ & & & 1.8 \\
$\psi_{25}$ & & & 4.8 \\
$\psi_{26}$ & & & 3.2 \\
$\psi_{27}$ & & & 1.2 \\
\hline
\end{tabular}

TABLE I: Contribution in percentage of each of the states to the oscillations in the density profile presented in figure 15.

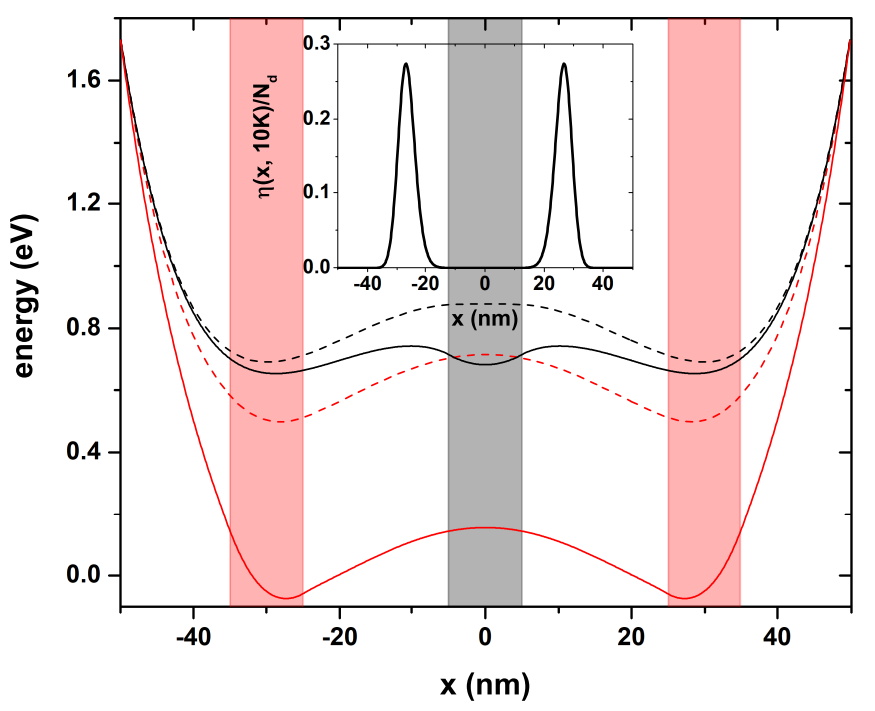

FIG. 16: Projection of the self-consistent potential in the $x$ direction considering two different doping zones, the black curves correspond to a central cylinder-shaped doping, as indicated by the dark vertical column, the red curves correspond to a cylindrical ring-shaped doping such as show the two light red columns. The dashed lines are for $N_{d}=1 \times 10^{18} \mathrm{~cm}^{-3}$ and the solid lines are for $N_{d}=5 \times 10^{18} \mathrm{~cm}^{-3}$. The inset shows the electron density obtained for $N_{d}=5 \times 10^{18} \mathrm{~cm}^{-3}$. Calculations are with $R_{0}=50 \mathrm{~nm}, r_{d}=5 \mathrm{~nm}$, and $T=10 \mathrm{~K}$.

Finally, in order to have a comparison about the effect of doping geometry on the self-consistent potential in the cylindrical Razavy-like QWR, Fig. 16 shows the projection of the self-consistent potential along the $x$ direction considering two different doping zones, the black curves correspond to a central cylinder-shaped doping, as indicated by the dark vertical column, the red curves correspond to a cylindrical ring-shaped doping (the two light red columns in the figure indicate the cross section of the ring in the $x z$ plane, the columns are centered at $x=-30 \mathrm{~nm}$ and $x=30 \mathrm{~nm}$ respectively). The dashed lines are for $N_{d}=1 \times 10^{18} \mathrm{~cm}^{-3}$ and the solid lines are for $N_{d}=5 \times 10^{18} \mathrm{~cm}^{-3}$. The inset shows the electron density obtained for $N_{d}=5 \times 10^{18} \mathrm{~cm}^{-3}$. Calculations are with $R_{0}=50 \mathrm{~nm}, r_{d}=5 \mathrm{~nm}$, and $T=10 \mathrm{~K}$. When the doping corresponds to the central cylinder, that is, to the black curves, one observes that when the density of donors in the system increases, there is a considerable decrease of the potential in the central zone. This is much more significant when the doping is included in a region with the shape of a cylindrical ring (red curves), where the potential is lowered by an average of $0.5 \mathrm{eV}$ in the regions between $-30 \mathrm{~nm}$ and $30 \mathrm{~nm}$. It should be noted that in the latter case there is no longer a specific decrease in the central area, that is, the repulsive character is not lost in the center of symmetry. Analyzing the continuous black curve it is possible to realize that, for higher donor densities, the dominant potential in the system will be the potential due to the redistribution of charges, that is, the Hartree potential and the Razavy potential loses significance. Therefore, when the density of donors accumulates in a central cylinder, the self-consistent electron density will be very sensitive to the magnitude of these donors since they can drastically modify the potential profile. The opposite case occurs when the donor density accumulates in a cylindrical ring. There, as the donor density increases, the dominant potential remains the Razavy one, and the Hartree potential only generates a decrease in magnitude of the potential without drastically modifying the shape. The inset in Fig. 16 shows the electron density for $N_{d}=5 \times 10^{18} \mathrm{~cm}^{-3}$ for the system with cylindrical ring doping. By comparing this result with the one presented in Fig. 12(b), we see a totally different profile in which the electrons accumulate mostly in the area of doped ring and, despite being at a low temperature, no Friedel-like oscillations are present. 


\section{CONCLUSIONS}

By using the effective mass and parabolic band approximations, the finite difference method, as well as a self-consistent calculation, we have investigated the features of total optical absorption coefficient of confined electrons in a delta-like doped Razavy-like quantum well under the combined effects of externally applied electric and magnetic field. In the absorption peaks, a clear blue shift is evidenced for all the transitions studied, keeping the donor density fixed, the shift being more significant for changes in the electric field than in the magnetic field, in all cases a change in the magnitude of the optical absorption peaks is presented. The transition with the greatest resistance to modifications due to external fields is $\alpha_{23}$. On the other hand, with the increase in donor density, red and blue shifts of the absorption peaks were also reported, as well as a decrease in their magnitude depending on the transition studied. This allows us to tune the system without modifying geometric parameters directly, only applying external fields or increasing the density of donors to obtain the maximum absorption of the material in the positions that are required for a certain application.

On the other hand, the delta-doping effect on electron states has been analyzed in quantum wire systems with exposed borders and circular cross-section subjected to an internal Razavy-like potential at low temperatures. Effects of varying geometric parameters such as the width of delta-doped layer, and not geometric as the density of donors in the system have been studied. In both cases a decrease in the magnitude of all electronic states has been found both with the increase of $r_{d}$ and $N_{d}$. The appearance of irregularities in the electron density profile has been reported for $r_{d}=10 \mathrm{~nm}$ and $15 \mathrm{~nm}$, these oscillations are Friedel-like and have been explained by means of the occupation of the electronic states of the system. We hope that this research will stimulate future investigations related to intentional doping in low-dimensional semiconductor heterostructures.

\section{Acknowledgments}

CAD is grateful to the Colombian Agencies: CODIUniversidad de Antioquia (Estrategia de Sostenibilidad de la Universidad de Antioquia and projects "Efectos de capas delta dopadas en pozos cuánticos como fotodetectores en el infrarrojo", "Propiedades magneto-ópticas y óptica no lineal en superredes de Grafeno", "Efectos ópticos intersubbanda, no lineales de segundo orden y dispersión Raman, en sistemas asimétricos de pozos cuánticos acoplados", and "Estudio de propiedades ópticas en sistemas semiconductores de dimensiones nanoscópicas"), and Facultad de Ciencias Exactas y Naturales-Universidad de Antioquia (CAD exclusive dedication project 2020-2021). CAD also acknowledges the financial support from El Patrimonio Autónomo
Fondo Nacional de Financiamiento para la Ciencia, la Tecnología y la Innovación Francisco José de Caldas (project: CD 111580863338, CT FP80740-173-2019). JAGC acknowledges the financial support from "University of Antioquia Doctoral Scholarship" in December 2020 , to the doctoral program, and to the postgraduate management of the University of Antioquia. MEMR acknowledges Mexican Conacyt for support through Grant A1-S-8218.

\section{Author contributions statement}

The contributions of the authors are as follows:

Hassen Dakhlaoui: proposed the problem and was responsible of the numerical calculations and writing of the manuscript.

J. A. Gil-Corrales: was responsible of the numerical calculations and writing of the manuscript.

A. L. Morales: participated in the discussion and writing of the manuscript.

E. Kasapoglu: was responsible of the numerical calculations and discussion.

A. Radu: participated in the discussion and writing of the manuscript.

R. Restrepo: participated in the discussion and writing of the manuscript.

V. Tulupenko: participated in the discussion and writing of the manuscript.

J. A. Vinasco: was responsible of the numerical calculations.

M. E. Mora-Ramos: participated in the discussion and writing of the manuscript.

C. A. Duque: was responsible of the numerical calculations, discussion, and writing of the manuscript.

\section{Competing interests statement}

The authors do not have any financial and non-financial competing interests statement. 
Data availability statement All the files with tables, figures, and codes are available. The corresponding au- thor will provide all the files in case they are requested.
[1] R. L. Restrepo, J. P. González-Pereira, E. Kasapoglu, A. L. Morales, and C. A. Duque, Linear and nonlinear optical properties in the terahertz regime for multiple-step quantum wells under intense laser field: electric and magnetic field effects, Opt. Mater. 86, 590-599 (2018).

[2] E. C. Niculescu, Electromagnetically induced transparency in an asymmetric double quantum well under non-resonant, intense laser fields, Opt. Mater. 64, 540-547 (2017).

[3] H. V. Phuc, N. Duy Anh Tuan, and L. Dinh, Linear and nonlinear magneto-optical absorption in a quantum well modulated by intense laser field, Superlattice Microst. 100, 1112-1119 (2016).

[4] J.-F. You, Q. Zhao, Z.-H. Zhang, J.-H. Yuan, K.-X. Guo, and E. Feddi, The effect of temperature, hydrostatic pressure and magnetic field on the nonlinear optical properties of AlGaAs/GaAs semi-parabolic quantum well, Int. J. Mod. Phys. B 33, 1950325 (12pp) (2019).

[5] G. Liu, R. Liu, G. Chen, Z. Zhang, K. Guo, and L. Lu, Nonlinear optical rectification and electronic structure in asymmetric coupled quantum wires, Results Phys. 17, 103027 (6pp) (2020).

[6] G. Liu, K. Guo, L. Xie, Z. Zhang, and L. Lu, Tunability of linear and nonlinear optical absorption in laterally-coupled $A l_{x} G a_{1-x} A s / G a A s$ quantum wires, J. Alloy. Compd. 746, 653-659 (2018).

[7] M. J. Karimi and M. Hosseini, Electric and magnetic field effects on the optical absorption of elliptical quantum wire, Superlattice Microst. 111, 96-102 (2017).

[8] E. Kasapoglu, C. A. Duque, M. E. Mora-Ramos, and I. Sökmen, The effects of the intense laser field on the nonlinear optical properties of a cylindrical $G a_{1-x} A l_{x} A s / G a A s$ quantum dot under applied electric field, Physica B 474, 15-20 (2015).

[9] M. G. Barseghyan, H. M. Baghramyan, A. A. Kirakosyan, and $\mathrm{D}$. Laroze, The transition from double to single quantum dot induced by THz laser field, Physica E 116, 113758 (4pp) (2020).

[10] A. Ghosh, A. Bera, and M. Ghosh, Modulating binding energy and interband emission energy of impurity doped quantum dots in presence of Gaussian white noise, Curr. Smart Mater. 2, 56-64 (2017).

[11] I. Karabulut and E. Paspalakis, The role of permanent dipoles on the intensity-dependent nonlinear optical properties in asymmetric coupled quantum wells under a static electric field, Physica E 81, 294-301 (2016).

[12] K. Li, K. Guo, X. Jiang, and M. Hu, Effect of positiondependent effective mass on nonlinear optical properties in a quantum well, Optik 132, 375-381 (2017).

[13] B. T. Diroll, M. Chen, I. Coropceanu, K. R. Williams, D. V. Talapin, P. Guyot-Sionnest, and R. D. Schaller, Polarized near-infrared intersubband absorptions in CdSe colloidal quantum wells, Nat. Commun. 10, 4511 (9pp) (2019).

[14] H. Dakhlaoui and M. Nefzi, Simultaneous effect of impurities, hydrostatic pressure, and applied potential on the optical absorptions in a GaAs field-effect transistor, Re- sults Phys. 15, 102618 (7pp) (2019).

[15] H. Noverola-Gamas, L. M. Gaggero-Sager, and O. Oubram, Interlayer distance effects on absorption coefficient and refraction index change in p-type double- $\delta$ doped GaAs quantum wells, Chinese Phys. B 28, 124207 (5pp) (2019).

[16] Q. Zhao, S. Aqiqi, J.-F. You, M. Kria, K.-X. Guo, E. Feddi, Z.-H. Zhang, and J.-H. Yuan, Influence of position-dependent effective mass on the nonlinear optical properties in $A l_{x} G a_{1-x} A s / G a A s$ single and double triangular quantum wells, Physica E 115, 113707 (7pp) (2020).

[17] Ri. Betancourt-Riera, Re. Betancourt-Riera, L. A. Ferrer-Moreno, and A. D. Sañu-Ginerte, Electron states and electron Raman scattering in a semiconductor quantum well with step-barriers: electric field effect, Physica B 575, 411700 (7pp) (2019).

[18] B. Amiri and A. Belghachi, First principles investigation of absorption coefficient in quantum well designed for solar cells, Optik 202, 163554 (6pp) (2020).

[19] H. Yildirim, Many-body effects on intersubband transitions in polar $\mathrm{ZnO} / \mathrm{ZnMgO}$ multiple quantum wells, Physica B 571, 26-31 (2019).

[20] Y.-Y. Chen, Y.-N. Li, R.-G. Wan, and H.-W. Yan, Tunable double-beam optical bistability in an asymmetric double quantum-well system, Phys. Lett. A 383, 125921 (7pp) (2019).

[21] S. Panda, T. Das, and B. K. Panda, Nonlinear optical susceptibilities in $\operatorname{In}_{x} G a_{1-x} N / G a N$ hexagonal single quantum well under applied electric field, Superlattice Microst. 135, 106238 (11pp) (2019).

[22] H. Dakhlaoui and M. Nefzi, Tuning the linear and nonlinear optical properties in double and triple $\delta$-doped GaAs semiconductor: Impact of electric and magnetic fields, Superlattice Microst. 136, 106292 (14pp) (2019).

[23] F. Ungan, M. K. Bahar, J. C. Martinez-Orozco, and M. E. Mora-Ramos, Optical responses in asymmetric hyperbolic-type quantum wells under the effect of external electromagnetic fields, Photonic. Nanostruct. 41, 100833 (5pp) (2020).

[24] N. D. Hien, C. A. Duque, E. Feddi, N. V. Hieu, H. D. Trien, L. T. T. Phuong, B. D. Hoi, L. T. Hoa, C. V. Nguyen, N. N. Hiev, and H. V. Phuc, Magneto-optical effect in GaAs/GaAlAs semi-parabolic quantum well, Thin Solid Films 682, 10-17 (2019).

[25] E. F. Schubert, Delta doping of III-V compound semiconductors: fundamentals and device applications, J. Vac. Sci. Technol. A 8, 2980-2996 (1990).

[26] K. Ploog, M. Hauser, and A. Fischer, Fundamental studies and device application of $\delta$-doping in GaAs Layers and in $A l_{x} G a_{1-x} A s / G a A s$ heterostructures, Appl. Phys. A 45, 233-244 (1988).

[27] A. C. Maciel, M. Tatham, J. F. Ryan, J. M. Worlock, R. E. Nahory, J. P. Harbison, and L. T. Forlez, Raman scattering from electronic excitations in periodically $\delta$-doped GaAs, Surf. Sci. 228, 251-254 (1990).

[28] L. Ioriatti, Thomas-Fermi theory of $\delta$-doped semicon- 
ductor structures: Exact analytical results in the highdensity limit, Phys. Rev. B 41, 8340-8344 (1990).

[29] J. C. Egues, J. C. Barbosa, A. C. Notari, P. Basmaji, L. Ioriatti, E. Ranz, and J. C. Portal, Electronic transport in periodically $\delta$-doped GaAs layers, J. Appl. Phys. 70, 3678-3680 (1991).

[30] M. H. Degani, Electron energy levels in a $\delta$-doped layer in GaAs, Phys. Rev. B 44, 5580-5584 (1991).

[31] M. H. Degani, Electronic properties of multiple Si $\delta$ doping in GaAs, J. Appl. Phys. 70, 4362-4365 (1991).

[32] J. Osvald, Electronic properties of a near surface $\mathrm{Si}$ $\delta$-doped GaAs under an applied electric field, J. Phys. D Appl. Phys. 37, 2655-2659 (2004).

[33] K.-M. Wong and D. W. E. Allsopp, Intersubband absorption modulation in coupled double quantum wells by external bias, Semicond. Sci. Tech. 24, 045018 (8pp) (2009).

[34] J. Krupski and M. Piętka, On the accuracy of the Thomas-Fermi-Dirac method applied to sub-band structure calculations in a $\delta$-doped semiconductor, Solid State Commun. 107, 141-144 (1998).

[35] E. Ozturk, Y. Ergun, H. Sari, and I. Sokmen, The selfconsistent calculation of Si $\delta$-doped GaAs structures, Appl. Phys. A 73, 749-754 (2001).

[36] E. Kasapoglu, F. Ungan, H. Sari, and I. Sökmen, The hydrostatic pressure and temperature effects on donor impurities in cylindrical quantum wire under the magnetic field, Physica E 42, 1623-1626 (2010).

[37] N. Raigoza, A. L. Morales, and C. A. Duque, Infinite potential barrier and hydrostatic pressure effects on impurity-related optical absorption spectra in GaAs double quantum wells, Braz. J. Phys. 36, 350-353 (2006).

[38] M. G. Barseghyan, A. A. Kirakosyan, and C. A. Duque, Hydrostatic pressure, electric and magnetic field effects on shallow donor impurity states and photoionization cross section in cylindrical GaAs-Ga $a_{1-x} A l_{x}$ As quantum dots, Phys. Status Solidi B 246, 626-629 (2009).

[39] I. Rodriguez-Vargas, M. E. Mora-Ramos, and C. A. Duque, Influence of the hydrostatic pressure onto the electronic and transport properties of n-type double $\delta$-doped GaAs quantum wells, Microelectr. J. 39, 438441 (2008).

[40] P. Nithiananthi and K. Jayakumar, Pressure study on the semiconductor-metal transition in a quantum well, Phys. Status Solidi B 246, 1238-1242 (2009).

[41] R. Khordad, S. Kheiryzadeh Khaneghah, and M. Masoumi, Effect of pressure on intersubband optical absorption coefficients and refractive index changes in a $V$ groove quantum wire, Superlattice Microst. 47, 538-549 (2010).

[42] L. M. Gaggero-Sager, G. G. Naumis, M. A. MuñozHernandez, and V. Montiel-Palma, Self-consistent calculation of transport properties in Si $\delta$-doped GaAs quantum wells as a function of the temperature, Physica B 405, 4267-4270 (2010).

[43] R. B. Dhafer, H. Saidi, and S. Ridene, Proposal of InP/AlInGaAs single delta quantum well for fiber-optic communications, Optik 158, 164-169 (2018).

[44] J. Osvald, Self-consistent analysis of Si $\delta$-doped layer placed in a non-central position in GaAs structure, Physica E 23, 147-151 (2004).

[45] M. Razavy, An exactly soluble Schrödinger equation with a bistable potential, Am. J. Phys. 48, 285 (1980).

[46] M. Baradaran and H. Panahi, Exact Solutions of a Class of Double-Well Potentials: Algebraic Bethe Ansatz, Adv.
High Energy Phys. 2017, 8429863 (2017).

[47] H. Karayer, D. Demirhan, K. G. Atman, Analytical exact solutions for the Razavy type potential, Math Meth Appl Sci. 93, 9185-9194 (2020).

[48] J. Feist, J. Galego, and F. J. García-Vidal, Polaritonic Chemistry with Organic Molecules, ACS Photonics 5, 205-216 (2018).

[49] Y. E. Panfil, D. Shamalia, J. Cui, S. Koley, and U. Banin, Electronic coupling in colloidal quantum dot molecules; the case of CdSe/CdS core/shell homodimers, J. Chem. Phys. 151, 224501 (2019).

[50] Z. Han, G. Czap, C. Xu, Chi-lun Chiang, D. Yuan, R. $\mathrm{Wu}$, and $\mathrm{W}$. Ho, Probing Intermolecular Coupled Vibrations between Two Molecules, Phys. Rev. Lett. 118, 036801 (2017).

[51] X. Li, A. Mandal, and P. Huo, Cavity frequencydependent theory for vibrational polariton chemistry, Nat. Commun. 12, 1315 (2021).

[52] E. Kasapoglu, H. Sari, I. Sökmen, J. A. Vinasco, D. Laroze, and C. A. Duque, Effects of intense laser field and position dependent effective mass in Razavy quantum wells and quantum dots, Physica E 126, 114461 (12pp) (2021).

[53] V. K. Arora, Quantum size effect in thin-wire transport. Phys. Rev. B 23, 5611-5612 (1981).

[54] G. W. Bryant, Hydrogenic impurity states in quantumwell wires. Phys. Rev. B 29, 6632-6639 (1984).

[55] S. Luryi and F. Capasso Resonant tunneling of twodimensional electrons through a quantum wire: A negative transconductance device. Appl. Phys. Lett. 47, 13471349 (1985); Erratum in 48, 1693 (1986).

[56] K. B. Wong, M. Jaros, and J. P. Hagon, Confined electron states in $G a A s-G a_{1-x} A l_{x} A s$ quantum wires. Phys. Rev. B 35, 2463-2466 (1987).

[57] T. Yamauchi, and Y. Arakawa, Tight binding analysis of GaAsAlGaAs quantum wire structures. Superlattices Microstruct. 10, 83-87 (1991).

[58] E. C. Garnett, M. L. Brongersma, Y. Cui, M. D. McGehee, Nanowire Solar Cells. Ann. Rev. Mater. Res. 41, 269-295 (1991).

[59] F. Zaouali, A. Bouazra, M. Said, A theoretical evaluation of optical properties of InAs/InP quantum wire with a dome cross-section. Optik, 174, 513-520 (2018).

[60] L. Van-Tan, T. V. Thang, N. D. Vy, H. T. Cao, Spin polarization and temperature dependence of electron effective mass in quantum wires. Phys. Lett. A, 383, 21102113 (2019).

[61] P. Hosseinpour, Effect of Gaussian impurity parameters on the valence and conduction subbands and thermodynamic quantities in a doped quantum wire. Solid State Commun. 322, 114061 (2020).

[62] B. D. Woods, S. D. Sarma, T. D. Stanescu, Subband occupation in semiconductor-superconductor nanowires. Phys. Rev. B 101, 045405 (2020).

[63] J.-B. Xia and W.-J. Fan, Electronic structures of superlattices under in-plane magnetic field, Phys. Rev. B 40, 8508-8515 (1989).

[64] K. F. Brennan and A. S. Brown, Theory of Modern Electronic Semiconductor Devices, John Wiley \& Sons, New York, 2002, p. 448.

[65] H. Dakhlaoui, The effects of doping layer location on the electronic and optical properties of GaN step quantum well, Superlattice Microst. 97, 439-447 (2016).

[66] H. B. Dakhlaoui and N. Mouna, Quantum size and mag- 
nesium composition effects on the optical absorption in the $M g_{x} Z n_{(1-x)} O / Z n O$ quantum well, Chem. Phys. Lett. 693, 40-45 (2018).

[67] H. Dakhlaoui and M. Nefzi, Enhancement of the optical absorption in $\mathrm{MgZnO} / \mathrm{ZnO}$ quantum well under external electric field, Optik 157, 1342-1349 (2018).

[68] M. K. Bahar, K.A. Rodríguez-Magdaleno, J.C. MartínezOrozco, M. E. Mora-Ramos, and F. Ungan, Optical properties of a triple AlGaAs/GaAs quantum well purported for quantum cascade laser active region, Mater. Today Commun. 26, 101936 (2021).

[69] A. S. Durmuslar, C. A. Billur, A. Turkoglu, and F. Ungan, Optical properties of a GaAs quantum well with new type of hyperbolic confinement potential: Effect of structure parameters and intense laser field, Opt. Commun. 499, 127266 (2021).

[70] A. Zangwill, Physics at Surfaces; Cambridge University Press: New York, NY, USA, p. 454 (1988).

[71] J. H. Luscombe, M. Luban, Lateral confinement in quantum nanostructures: Self-consistent screening potentials, Appl. Phys. Lett. 57, 61-63 (1990).

[72] K. Pieniak, M. Chlipala, H. Turski, W. Trzeciakowski, G. Muziol, G. Staszczak, A. Kafar, I. Makarowa, E.
Grzanka, S. Grzanka, C. Skierbiszewski, and T. Suski, Quantum-confined Stark effect and mechanisms of its screening in InGaN/GaN light-emitting diodes with a tunnel junction, Opt. Express 29, 1824-1837 (2021).

[73] B.N. Aneeshkumar, Independent control of valence- and conduction-band states in composite quantum wells, Phd Thesis Eindhoven University of Technology 29 (2004).

[74] D. Makhlouf, M. Choubani, F. Saidi, and H. Maaref, Applied electric and magnetic fields effects on the nonlinear optical rectification and the carrier's transition lifetime in InAs/GaAs core/shell quantum dot, Mater. Chem. Phys. 267, 124660 (2021).

[75] A. Surrente, M. Baranowski, and P. Plochocka, Perspective on the physics of twodimensional perovskites in high magnetic field, Appl. Phys. Lett. 118, 170501 (2021).

[76] A. Baydin, T. Makihara, N. M. Peraca, and J. Kono, Time-domain terahertz spectroscopy in high magnetic fields, Front. Optoelectron. 14, 110-129 (2021).

[77] A. Arora, Magneto-optics of layered two-dimensional semiconductors and heterostructures: Progress and prospects, J. Appl. Phys. 129, 120902 (2021). 